\title{
K3 surfaces with a non-symplectic automorphism and product-quotient surfaces with cyclic groups
}

\author{
Alice Garbagnati and Matteo Penegini
}

\begin{abstract}
We classify all the K3 surfaces which are minimal models of the quotient of the product of two curves $C_{1} \times C_{2}$ by the diagonal action of either the group $\mathbb{Z} / p \mathbb{Z}$ or the group $\mathbb{Z} / 2 p \mathbb{Z}$ where $p$ is an odd prime. These K3 surfaces admit a non-symplectic automorphism of order $p$ induced by an automorphism of one of the curves $C_{1}$ or $C_{2}$. We prove that most of the K3 surfaces admitting a non-symplectic automorphism of order $p$ (and in fact a maximal irreducible component of the moduli space of K3 surfaces with a non-symplectic automorphism of order $p$ ) are obtained in this way.

In addition, we show that one can obtain the same set of K3 surfaces under more restrictive assumptions namely one of the two curves, say $C_{2}$, is isomorphic to a rigid hyperelliptic curve with an automorphism $\delta_{p}$ of order $p$ and the automorphism of the K3 surface is induced by $\delta_{p}$.

Finally, we describe the variation of the Hodge structures of the surfaces constructed and we give an equation for some of them.
\end{abstract}

\section{Introduction}

One of the main themes of interest in the study of K3 surfaces $S$ regards their automorphisms. We call an automorphism $\mathrm{g}$ of $S$ non-symplectic if it acts nontrivially on the nowhere vanishing holomorphic 2 -form $\omega$. In the case $|\mathbf{g}|=p$, a prime number, $\mathbf{g}(\omega)=\zeta_{p} \omega$, where $\zeta_{p}$ is a primitive $p$-th root of unity. The pairs $(S, \mathbf{g})$ are quite rare, in the sense that there are strict restrictions on both $p$, which must be smaller than or equal to 19 , and on the K3 surface $S$, which cannot be generic in the moduli space. More precisely, the families of K3 surfaces with a nonsymplectic automorphism of odd prime order have a finite number of connected components and the biggest of them has dimension 9 while the moduli space of the K3 surfaces is 20-dimensional.

Several authors worked on the classification of K3 surfaces admitting a nonsymplectic automorphism of odd prime order (see, e.g., [1], [35], [2], [20], [30], [29], 
and [28]), and their complete classification was given in [2]. The classification is based on the following procedure: first, a non-symplectic automorphism $\mathbf{g}$ of order $p$ acting on a K3 surface $S$ determines an action on the lattice $T:=$ $\left.\left(H^{2}(S, \mathbb{Z})^{\mathbf{g}}\right)^{\perp}\right) \subset H^{2}(S, \mathbb{Z})$. The lattice $T$ satisfies several conditions. Second, one lists all the lattices with such conditions. Third, for each lattice $T$ in the list one has to actually construct an example of a K3 surface admitting a non-symplectic automorphism $\mathbf{g}$ and such that $T \simeq\left(H^{2}(S, \mathbb{Z})^{\mathbf{g}}\right)^{\perp}$. Each example is given by an ad hoc construction.

The aim of this paper is to give a systematical way to construct most of these K3 surfaces by showing that they are the minimal models of product-quotient surfaces (i.e., of the minimal resolution of the quotient $\left(C_{1} \times C_{2}\right) / G$, where the $C_{i}$ are curves of genus $g(C) \geq 1$ and $G$ is a finite group acting diagonally on the product, see Definition 3.2). In addition, as we have already observed, the pairs $(S, \mathbf{g})$ are quite "special", we give here a geometrical interpretation of this "speciality" in many cases: $S$ is the minimal model of a product-quotient surface. Moreover, we prove that there is a curve which seems to play a central rôle in this construction. For each odd prime $p$, we define the curve $D_{p}$ as the hyperelliptic curve with equation $v^{2}=u^{p}+1$. It clearly admits an automorphism $\delta_{p}$ of order $p$, acting on $u$ as the multiplication by $\zeta_{p}$, and an automorphim $\tau_{p}$ of order $2 p$ which is the composition of $\delta_{p}$ with the hyperelliptic involution. We prove the following theorem.

Theorem 1.1. If $S$ is a $K 3$ surface admitting a non-symplectic automorphism of order $p=3$ (resp. $p=5,7,11,13$, resp. $p=17,19$ ) whose fixed locus contains at least 2 (resp. 1, resp. 0) curves and which acts trivially on the Picard group, then it is the minimal model of a resolution of the quotient $\left(C_{1} \times D_{p}\right) /\left(\mathbf{g}_{1} \times \tau_{p}\right)$, where $\mathbf{g}_{1}$ is an automorphism of $C_{1}$ of order $2 p$. The non-symplectic automorphism of order $p$ on $S$ is induced by the automorphism id $\times \delta_{\mathrm{p}}$.

The condition on the action of the automorphism on the Picard group assures that the K3 surface is generic among the ones with a non-symplectic automorphism with the required fixed locus.

Any K3 surface $S$ constructed in the Theorem 1.1 admits two isotrivial families of curves, whose general member is isomorphic to $C_{1}$ and $D_{p}$ respectively. The non-symplectic automorphism of order $p$ on $S$ is in fact given by the action of an automorphism on each member of one of these families.

We observe that the K3 surfaces given by the Theorem 1.1 admit a nonsymplectic automorphism of order $2 p$ too, the one induced by id $\times \tau_{\mathrm{p}}$. This gives a geometrical explanation of the following significative result on non-symplectic automorphisms: if a K3 surface $S$ admits a non-symplectic automorphism of order $p$, under certain conditions on the fixed locus it admits in fact a purely non-symplectic automorphism of order $2 p$ (see Theorems 1.6 and 1.7 in [15], and [8], for the precise statement).

The proof of the Theorem 1.1 is based on the construction of the K3 surfaces that we now explain. We first bound the genus of the curves $C$ with a cyclic group of automorphisms $G$ of order $p$ (resp. $2 p$ ), having the properties that $C / G \simeq \mathbb{P}^{1}$, and such that there exists an eigenspace $H^{1,0}(C)_{\zeta_{p}^{i}}\left(\right.$ resp. $\left.H^{1,0}(C)_{-\zeta_{p}^{i}}\right)$ of dimen- 
sion 1 of the induced action. This is achieved by exploiting the Riemann existence theorem, the holomorphic Lefschetz fixed-point formula, and the Chevalley-Weil formula. Second, we classify all these curves and we couple them by choosing the action of $G$ in such a way that the singular surfaces $\left(C_{1} \times C_{2}\right) / G$ have $p_{g}=1$ and $q=0$. Third, we resolve the singularities and we get product-quotient surfaces $X$ which are not minimal models, but in two cases. We observe that $K_{X}^{2}$ could be very negative. After having found all the $(-1)$-curve on $X$ (this is a quite delicate task, see e.g., [5]) we carefully contract them to produce a minimal model $S$ of $\left(C_{1} \times C_{2}\right) / G$. Finally, we prove that $S$ is a K3 surface.

As a byproduct of the proof we classify the K3 surfaces which are minimal models of the product-quotient surfaces with the groups $\mathbb{Z} / p \mathbb{Z}$ and $\mathbb{Z} / 2 p \mathbb{Z}$. Hence, we obtain also a "negative" result: if a K3 surface does not satisfy the hypothesis of Theorem 1.1 (i.e., either it does not admit a non-symplectic automorphism of order $p$, or it admits a non-symplectic automorphism of order $p$, but its fixed locus does not satisfy the condition of the Theorem 1.1), then this K3 surface is not the minimal model of a product-quotient with group $\mathbb{Z} / 2 p \mathbb{Z}$. However, we cannot exclude that such a K3 surface is the minimal model of a product-quotient with a different group. Indeed, we are aware that this is the case for at least certain families of K3 surfaces admitting a non-symplectic automorphism of order 3 . We shall analyze this problem in a forthcoming article.

This paper is organized as follows.

In Section 2 we briefly recall classical results on cyclic covers of $\mathbb{P}^{1}$. We establish the upper and lower bound for the genus of a curve $C$ with a cyclic group of automorphisms of odd prime order, and the property that there exists an eigenspace $H^{1,0}(C)_{\zeta_{p}^{i}}$ of dimension 1 . We state similar results for cyclic groups of automorphisms of order $2 p$. Moreover, we give some explanatory examples introducing the curve $D_{p}$.

Section 3 is divided into three parts. In the first part we give the definition of product-quotient surfaces and we recall the properties of these surfaces that are needed for our purposes. In the second part we calculate the Hodge numbers of product-quotient surfaces. In the last subsection we describe some automorphisms of the minimal model of a product-quotient surface.

In Section 4 we describe first the procedure we used to construct productquotient surfaces with group either $\mathbb{Z} / p \mathbb{Z}$ or $\mathbb{Z} / 2 p \mathbb{Z}$ and $p_{g}=1$ and $q=0$; and second, we give a method to prove that these surfaces are K3 surfaces.

Sections 5 and 6 present the main results of the paper on K3 surfaces which are minimal models of product-quotient surfaces with group either $\mathbb{Z} / p \mathbb{Z}$ or $\mathbb{Z} / 2 p \mathbb{Z}$ respectively. Moreover, in these two sections one can find the tables with the surfaces we constructed (see Table 1 and Table 2).

In Section 7 we give the equations for the singular models of the K3 surfaces constructed for $p=3,5,7,11$. In particular if $p=5,7,11$ we describe these surfaces as hypersurfaces in weighted projective spaces.

In the last section we describe the variation of the Hodge structures of the K3 surfaces constructed in terms of the Hodge structure of $H^{1}\left(C_{1}\right)$ relating them by a half twist. 
The Magma [22] code used in our calculations can be found at:

users.mat .unimi.it/users/penegini/publications/programmaToT_v7.m

There are essentially two programs, Surfacesp and t1t2PtsSurfaces. The former gives a list of all product-quotient surfaces $X$ with group $\mathbb{Z} / p \mathbb{Z}$ ( $p$ any odd prime), $p_{g}(X)=1$ and $q(X)=0$, as well as the singularities of $\left(C_{1} \times C_{2}\right) /\left(\mathbf{g}_{1} \times \mathbf{g}_{\mathbf{2}}\right)$, this program becomes very slow as $p$ increases. The latter program gives a similar list, it is much faster, it works also for the group $\mathbb{Z} / 2 p \mathbb{Z}$, but it requires two additional data which are the number of ramification points of the two coverings $C_{i} \rightarrow C_{i} / G$.

Notation. We work over the field of complex numbers $\mathbb{C}$.

Unless otherwise stated, $p$ will be always an odd prime number.

We will denote by $\zeta_{n}:=e^{2 \pi i / n}$ a primitive $n$-th root of unity.

By "curve" or "Riemann surface" we mean a projective, non-singular curve $C$. We denote by $H^{1,0}(C)=H^{0}\left(C, \Omega_{C}^{1}\right)$ and by

$$
g(C):=h^{1,0}(C):=\operatorname{dim} H^{1,0}(C)
$$

the genus of the curve.

By "surface" we mean a projective, non-singular surface $S$, and for such a surface,

$$
\omega_{S}=\mathcal{O}_{S}\left(K_{S}\right)
$$

denotes the canonical class,

$$
p_{g}(S)=h^{2,0}(S)=h^{0}\left(S, \omega_{S}\right)
$$

is the geometric genus,

$$
q(S)=h^{1,0}(S)=h^{1}\left(S, \omega_{S}\right)
$$

is the irregularity and

$$
\chi(S)=1-q(S)+p_{g}(S)
$$

is the Euler-Poincaré characteristic. The Noether formula is

$$
12 \chi(S)=K_{S}^{2}+e(S)
$$

where $e(S)$ is the Euler number of $S$.

By abuse of notation by "(-1)-curve" we mean a curve $C$ with $C \simeq \mathbb{P}^{1}$ and $C^{2}=-1$.

Acknowledgments: We warmly thanks Bert van Geemen and Roberto Pignatelli for several essential suggestions and useful discussions. We also thank Ciro Ciliberto, Shigeyuki Kondō, Rita Pardini and Francesco Polizzi for their comments, and the anonymous referees whose valuable comments helped us to improve the exposition. 


\section{Curves}

This section is devoted to recall some classical results on maps between curves and in particular on cyclic covers of $\mathbb{P}^{1}$ (see for example Chapter 2 in [32]). These results are well known but essential for the understanding of the Magma program used in the calculations. We also provide examples of curves which will be used in the rest of the paper (see Example 2.5).

\subsection{The Riemann existence theorem}

We consider a cyclic cover $C \rightarrow \mathbb{P}^{1}$. As a consequence of the Riemann existence theorem (see Chapter III in [24]), we have the following.

Proposition 2.1 (Corollary 4.10 in [24]). Let $G:=\langle x\rangle$ be isomorphic to $\mathbb{Z} / n \mathbb{Z}$. Fix a finite set $B:=\left\{b_{1}, \ldots, b_{r}\right\} \subset \mathbb{P}^{1}$ of points. Then there is a $1: 1$ correspondence

$$
\begin{gathered}
\left\{\begin{array}{c}
\text { Isomorphism classes of cyclic covers } \\
\pi: C \rightarrow \mathbb{P}^{1} \text { of degree } n \\
\text { whose branch points lie in } B
\end{array}\right\} \\
\uparrow 1: 1 \\
\left\{\begin{array}{c}
r \text {-tuples }\left(x^{\xi_{1}}, \ldots, x^{\xi_{r}}\right), \text { of generators of } G, \xi_{i} \in \mathbb{Z} / n \mathbb{Z} \\
\text { such that } \sum_{i=1}^{r} \xi_{i} \equiv 0 \bmod n \\
\text { up to Hurwitz moves }
\end{array}\right\} .
\end{gathered}
$$

Denote by $m_{i}$ the branching number relative to the point $b_{i}$ in the cover $f: C \rightarrow \mathbb{P}^{1}$ associated to $B$. The genus $g(C)$ is determined by the Riemann-Hurwitz relation

$$
2 g(C)-2=|G|\left(-2+\sum_{i=1}^{r}\left(1-\frac{1}{m_{i}}\right)\right) .
$$

Definition 2.2 (Definition 0.8 in [5]). A representative of a Hurwitz equivalence class of $r$-tuples associated to a $G$-cover of $\mathbb{P}^{1}$ is called a spherical system of generators for $\mathbb{Z} / n \mathbb{Z}$.

As an automorphism group of $C, G=\langle\mathbf{g}\rangle$ gives a conformal self-mapping g: $C \rightarrow C$ of order $n$. Please notice that the abstract group $G$ has also other realizations: as image of an admissible epimorphism $\langle x\rangle$ or as local action $\left\langle\zeta_{n}\right\rangle$ near a point $P \in C$. Suppose that $\mathrm{g}^{k}$ fixes a point $P \in C$, then in a suitable local coordinate $z$ near $P$ we must have $\mathbf{g}^{k}(z)=\zeta_{n}^{i} z$. Thus $\mathbf{g}^{k}$ is locally a rotation at $P$ and the rotation angle is determined in the following proposition.

Proposition 2.3 (Theorem 7 in [19]). Let $C$ be a curve associated to the spherical system of generators $\left(x^{\xi_{1}}, \ldots, x^{\xi_{r}}\right)$. Let $P_{j}$ be a point with a non trivial stabilizer in $\langle\mathbf{g}\rangle \subset \operatorname{Aut}(C)$ of order d generated by $\mathbf{g}^{\xi_{j}}$. Then $\mathbf{g}^{\xi_{j}}(z)=\zeta_{n}^{\eta_{j}} z$ where $z$ is a local coordinate near the point $P_{j}$ and $\xi_{j} \eta_{j} \equiv n / d \bmod n$ and $0<\eta_{j}<d$. 
If $n=p$ is a prime number, we denote by $a_{i}, i=1, \ldots, p-1$, the number of ramification points of $C$ where the local action is given by $\zeta_{p}^{i}$. If $n=2 p$, with $p$ an odd prime number, we denote by $a_{i}, i=1, \ldots p-1$ the number of total ramification points of $C$ where the local action is given by $-\left(\zeta_{p}^{i}\right)$, by $a_{j}, j=p, \ldots, 2 p-2$ the number of points with stabilizer generated by $\mathrm{g}^{2}$ and such that the local action of $\mathbf{g}^{2}$ is $\zeta_{p}^{j}$, and finally by $a_{2 p-1}$ the number of points whose stabilizer is generated by $\mathbf{g}^{p}$.

Let $\mathbb{Z} / n \mathbb{Z} \simeq G \subset \operatorname{Aut}(C)$, then there is an induced linear action of $G$ on the Dolbeault cohomology $H^{*, *}(C)$. Let us denote by $\mathbf{g}$ a chosen generator of $G$. Then $\mathbf{g}^{*}$ acts on $H^{0,1}(C)$, and the dimension of each eigenspace will be denoted by

$$
\alpha_{j}:=\operatorname{dim} H^{0,1}(C)_{\zeta_{n}^{j}} .
$$

By the Chevalley-Weil formula ([7]) (or equivalently by the application of the Lefschetz holomorphic formula [18], page 426 , to $\mathbf{g}^{j}$ ) one can deduce the explicit relation among the $a_{i}$ 's and the $\alpha_{j}$ 's. This formula is particularly easy in case $n=p$, and is the following:

$$
\alpha_{p-r}=-1+\frac{1}{p} \sum_{l=1}^{p-1} a_{l} l^{-1}(p-r),
$$

where the product $l^{-1}(p-r)$ is taken $\bmod p$, and $r=1, \ldots, n-1$.

In case $n=2 p$ we refer to the Magma program (function MaxGenus). In both cases, a trivial consequence is the following corollary.

Corollary 2.4. Let $C \rightarrow \mathbb{P}^{1}$ be a $G$ cover and $r$ be the number of ramification points for $\pi: C \rightarrow \mathbb{P}^{1}$.

If $G \simeq \mathbb{Z} / p \mathbb{Z}$ and there exists $i \in\{1, \ldots, p-1\}$ such that $\alpha_{i}=1$, then $(p-1) / 2 \leq g(C) \leq(p-1)^{2}$ and $3 \leq r \leq 2 p$.

If $G \simeq \mathbb{Z} / 2 p \mathbb{Z}$ and there exists $i \in\{1, \ldots, p-1\}$ such that $\operatorname{dim}\left(H^{0,1}(C)_{-\zeta_{p}^{i}}\right)$ $=1$, then $(p-1) / 2 \leq g(C) \leq(2 p-1)^{2}$ and $3 \leq r \leq 4 p$.

Example 2.5. Let $G \simeq \mathbb{Z} / p \mathbb{Z}$. The triple $\left(x^{p-1}, x^{p-1}, x^{2}\right)$ is a spherical system of generators for $G$. Hence, by Theorem 2.1 there exists a curve, $D_{p}$, such that $D_{p}$ is a $p: 1$ cover of $\mathbb{P}^{1}$ branched in 3 points and the cover automorphism $\delta_{p}$ acts locally as $\zeta_{p}^{p-1}$ near two fixed points and as $\zeta_{p}^{(p+1) / 2}$ near the other one. This means that the only non zero $a_{i}$ 's are $a_{p-1}=2$ and $a_{(p+1) / 2}=1$. For every choice of three points in $\mathbb{P}^{1}$ there exists an involution of $\mathbb{P}^{1}$ switching the first two points and fixing the other, so there exists an involution of $\mathbb{P}^{1}$ acting in this way on the branch points of $D_{p} \rightarrow \mathbb{P}^{1}$. This induces an involution on $D_{p}$, which will be denoted by $\iota_{p}$. We observe that $\iota_{p} \delta_{p}=\delta_{p} \iota_{p}$ and we denote by $\tau_{p}=\iota_{p} \delta_{p}$. An equation of $D_{p}$ and the corresponding equation for its automorphisms are:

$$
\begin{array}{rlrl}
D_{p}: u^{p}=v^{2}-1, & \delta_{p}:(u, v) & \mapsto\left(\zeta_{p}^{p-1} u, v\right), \\
\iota_{p}:(u, v) \mapsto(u,-v), & \tau_{p}:(u, v) \mapsto\left(\zeta_{p}^{p-1} u,-v\right) .
\end{array}
$$


The genus of $D_{p}$, computed by the Riemann-Hurwitz formula, is $g\left(D_{p}\right)=(p-$ $1) / 2$. The curve $D_{p}$ is hyperelliptic over $\mathbb{P}_{[u]}^{1}$ and so a basis for $H^{1,0}\left(D_{p}\right)$ is given by $\left\{u^{j} d u / v\right\}, j=0, \ldots,(p-3) / 2$. Therefore the eigenspaces decomposition of $H^{0,1}\left(D_{p}\right)=\overline{H^{1,0}\left(D_{p}\right)}$ for the induced action of $\delta_{p}$ is $\alpha_{i}=1$ if $i=1, \ldots,(p-1) / 2$ and $\alpha_{i}=0$ if $i=(p+1) / 2, \ldots, p-1$.

The automorphism $\tau_{p}$ of order $2 p$ is associated to a spherical system of generators $\left(x^{p+2}, x^{2 p-2}, x^{p}\right)$. The action of $\tau_{p}$ on the form $u^{i} d u / v$ is the multiplication by $-\zeta_{p}^{i+1}, 0 \leq i \leq(p-3) / 2$. Hence, the eigenspaces decomposition of $H^{1,0}\left(D_{p}\right)$ for $\tau_{p}$ is the following: if $0 \leq i \leq(p-3) / 2$ and $i \equiv 0 \bmod 2$, then $\alpha_{i+1}=1$; if $0 \leq i \leq(p-3) / 2$ and $i \equiv 1 \bmod 2$, then $\alpha_{p+i+1}=1 ; \alpha_{j}=0$ otherwise. We observe that $\tau_{p}$ switches two of the fixed points of $\delta_{p}$ and fixes the third fixed point of $\delta_{p}$. Clearly the two points switched by $\tau_{p}$ are those where the local action of $\delta_{p}$ is $\zeta_{p}^{p-1}$, and the point fixed both by $\tau_{p}$ and by $\delta_{p}$ is the one where the local action of $\delta_{p}$ is $\zeta_{p}^{(p+1) / 2}$. The automorphism $\tau_{p}^{2}$ coincides with $\delta_{p}^{2}$, and this identifies the local action of $\tau_{p}$ : $\tau_{p}$ fixes one point with local action $-\zeta_{p}^{(p+1) / 2} ; \tau_{p}^{2}$ fixes other two points (switched by $\tau_{p}$ ) with local action $\zeta_{p}^{p-2} ; \tau_{p}^{p}$ fixes other $p$ points (permuted by $\tau_{p}$ ) with local action $-\zeta_{p}^{p}=-1$.

Finally we observe that $D_{3}$ is the elliptic curve with complex multiplication of order 3 , which is associated to the automorphism $\delta_{3}$.

\section{Surfaces}

In this section we recall the properties of the product-quotient surfaces and their minimal models and we calculate their numerical invariants.

\subsection{Product-quotient surfaces}

Let us consider two curves $C_{1}$ and $C_{2}$ of genera greater than or equal to 1 , and their product $C_{1} \times C_{2}$. Then $\operatorname{Aut}\left(\mathrm{C}_{1}\right) \times \operatorname{Aut}\left(\mathrm{C}_{2}\right) \subset \operatorname{Aut}\left(\mathrm{C}_{1} \times \mathrm{C}_{2}\right)$. One can say even more in the case $g\left(C_{i}\right) \geq 2$.

Lemma 3.1 (Corollary 3.9 in [6]). Let us assume $g\left(C_{1}\right) \geq 2$ and $g\left(C_{2}\right) \geq 2$. If $C_{1} \neq C_{2}$, then $\operatorname{Aut}\left(\mathrm{C}_{1} \times \mathrm{C}_{2}\right)=\operatorname{Aut}\left(\mathrm{C}_{1}\right) \times \operatorname{Aut}\left(\mathrm{C}_{2}\right)$; otherwise, $\operatorname{Aut}\left(\mathrm{C}_{1} \times \mathrm{C}_{2}\right)=$ $\left(\operatorname{Aut}\left(\mathrm{C}_{1}\right) \times \operatorname{Aut}\left(\mathrm{C}_{2}\right)\right) \rtimes \mathbb{Z} / 2 \mathbb{Z}$.

Let $G \subset \operatorname{Aut}\left(\mathrm{C}_{1}\right) \times \operatorname{Aut}\left(\mathrm{C}_{2}\right)$ be a finite group and consider $\left(C_{1} \times C_{2}\right) / G$, where $G$ acts diagonally on the product $C_{1} \times C_{2}$.

Definition 3.2. The minimal resolution $X$ of the singularities of $\left(C_{1} \times C_{2}\right) / G$, where $G$ is a finite group with a diagonal action on the direct product of two smooth curves $C_{1}$ and $C_{2}$ of respective genera at least 1 , is called a product-quotient surface with group $G$.

We call $\left(C_{1} \times C_{2}\right) / G$ the quotient model of the product-quotient surface $X$.

Product-quotient surfaces were intensively studied; we refer to [5], [26] and [4] for a detailed account. We recall only some facts important for our purposes. 
Remark 3.3. The following facts hold:

1) There are only finitely many points on $C_{1} \times C_{2}$ with non trivial stabilizer, which is cyclic. Therefore the quotient model has only a finite number of cyclic quotient singularities.

A cyclic quotient singularity is locally analytic isomorphic to the quotient of $\mathbb{C}^{2}$ by the diagonal linear automorphism with eigenvalues $e^{2 \pi i / n}, e^{2 \pi i q / n}$, with $(q, n)=1$. This singularity is called of type $\frac{1}{n}(1, q)$, or $q / n$ for short (see Remark 1.1 in $[5])$.

2) The exceptional divisor $E$ on the minimal resolution of a cyclic quotient singularity is given by a Hirzebruch-Jung string (see e.g., Chapter III, Section 5 in [3]). A Hirzebruch-Jung string (HJ-string, for short) is a union $\widetilde{E}:=\cup_{i}^{k} E_{i}$ of smooth rational curves $E_{i}$ such that:

- $E_{i}^{2}=-b_{i} \leq-2$ for all $i$,

- $E_{i} E_{j}=1$ if $|i-j|=1$,

- $E_{i} E_{j}=0$ if $|i-j| \geq 2$,

where the $b_{i}$ 's are given by the continued fraction associated to $\frac{1}{n}(1, q)$. Indeed, by the formula:

$$
\frac{n}{q}=b_{1}-\frac{1}{b_{2}-\frac{1}{\cdots-\frac{1}{b_{k}}}} .
$$

3) A product-quotient surface comes together with two isotrivial fibrations. Let us consider one of them: $\pi_{2}: X \rightarrow C_{2} / G$. Take any point $b \in C_{2} / G$, and let $F$ denote the fibre of $\pi_{2}$ over $b$. Then (see Theorem 2.1 in [33]):

- The reduced structure of $F$ is the union of an irreducible smooth curve $Y$, called the central component of $F$, and either none or at least two mutually disjoint HJ-strings, each one meeting $Y$ at one point. These strings are in one-to-one correspondence with the branch points of $C_{1} \rightarrow\left(C_{1} / H\right)$, where $H \subset G$ is the stabilizer of $b$.

- The intersection of a string with $Y$ is transversal and it takes place at only one of the end component of the string.

4) There are formulae for calculating the self intersection of the canonical divisor of a product-quotient surface:

$$
K_{X}^{2} \geq \frac{8\left(g\left(C_{1}\right)-1\right)\left(g\left(C_{2}\right)-1\right)}{|G|}+\sum_{x \in \operatorname{Sing}(X)} h_{x},
$$

where $h_{x}$ depends on the type of singularity at $x$ and the equality holds if $g\left(C_{i}\right) \geq 2$, for at least one value of $i \in\{1,2\}$. If $x$ is a cyclic quotient singularity of type $\frac{1}{n}(1, q)$, then

$$
h_{x}:=2-\frac{2+q+q^{\prime}}{n}-\sum_{i=1}^{k}\left(b_{i}-2\right),
$$

where $q^{\prime} \in\{1, \ldots, n-1\}$ is such that $q q^{\prime} \equiv 1 \bmod n$, and $\left[b_{1}, \ldots, b_{k}\right]$ is the continued fraction associated to $\frac{1}{n}(1, q)$ (see Remark 1.1 in [5]). 
5) Finally, notice that $X$ is not necessarily a minimal model. Indeed, we will treat mostly examples with $X$ not minimal.

\subsection{Hodge structure of $X$}

Sometimes it is useful to keep track of the action of an automorphism on each curve separately; that is why in the following we assume $G \simeq \mathbb{Z} / n \mathbb{Z} \simeq\langle x\rangle \hookrightarrow \operatorname{Aut}\left(\mathrm{C}_{1}\right)$ $x \mapsto \mathbf{g}_{1}$, and $G \hookrightarrow \operatorname{Aut}\left(\mathrm{C}_{2}\right), x \mapsto \mathbf{g}_{2}$. We have $G=\left\langle\mathrm{g}_{1} \times \mathbf{g}_{2}\right\rangle \subset \operatorname{Aut}\left(\mathrm{C}_{1} \times \mathrm{C}_{2}\right)$, and we write $\left(C_{1} \times C_{2}\right) /\left(\mathbf{g}_{1} \times \mathbf{g}_{2}\right)$ for $\left(C_{1} \times C_{2}\right) /\left(\left\langle\mathbf{g}_{1} \times \mathbf{g}_{2}\right\rangle\right)$.

We shall describe the Hodge structure of the product-quotient surface $X$ whose quotient model is $\left(C_{1} \times C_{2}\right) / G$. The Hodge numbers of $X$ are determined by the action of $G$ on the cohomology of $C_{1}$ and $C_{2}$.

As in the previous section, we denote by $\alpha_{i}, i=1, \ldots, n$ the dimension of the eigenspace $H^{1,0}\left(C_{1}\right)_{\zeta_{n}^{i}}$ with eigenvalue $\zeta_{n}^{i}$ w.r.t. the action of $\mathbf{g}_{1}$ and by $\beta_{i}$, $i=1, \ldots, n$, the dimension of the eigenspace $H^{1,0}\left(C_{2}\right)_{\zeta_{n}^{i}}$ with eigenvalue $\zeta_{n}^{i}$ with respect to the action of $\mathbf{g}_{\mathbf{2}}$.

By Satz 1 in [13], we have

$$
H^{0}\left(X, \Omega_{X}^{i}\right) \simeq H^{0}\left(C_{1} \times C_{2}, \Omega_{C_{1} \times C_{2}}^{i}\right)^{G} .
$$

with $i=0,1,2$

Thus by the Künneth formula, we have

- $H^{0,0}(X)=H^{0,0}\left(C_{1} \times C_{2}\right)^{G}=H^{0,0}\left(C_{1}\right) \otimes H^{0,0}\left(C_{2}\right)$.

- $H^{1,0}(X)=H^{1,0}\left(C_{1} \times C_{2}\right)^{G}=H^{1,0}\left(C_{1}\right)_{\mathrm{id}} \otimes H^{0,0}\left(C_{2}\right) \oplus H^{0,0}\left(C_{1}\right) \otimes H^{1,0}\left(C_{2}\right)_{\mathrm{id}}$; in particular, $h^{1,0}(X)=h^{0,1}(X)=\alpha_{n}+\beta_{n}$.

- $H^{2,0}(X)=H^{2,0}\left(C_{1} \times C_{2}\right)^{G}=\sum_{i=1}^{n} H^{1,0}\left(C_{1}\right)_{\zeta_{n}^{i}} \otimes H^{1,0}\left(C_{2}\right)_{\zeta_{n}^{n-i}}$; in particular, $h^{2,0}(X)=h^{0,2}(X)=\sum_{i=1}^{n} \alpha_{i} \beta_{n-i}$.

In order to find $h^{1,1}(X)$, one has to know the number and the type of singularities of $\left(C_{1} \times C_{2}\right) / G$. Indeed, the desingularization of $\left(C_{1} \times C_{2}\right) / G$ introduces some exceptional divisors which increase the Picard number of the surface and thus $h^{1,1}(X)$.

Here we describe the structure of $H^{1,1}(X)$ starting from the description of the action of $\mathbf{g}_{\mathbf{i}}$ on $C_{i}, i=1,2$.

Let us consider the set of points of $C_{1}$ (resp. $C_{2}$ ) with a non trivial stabilizer with respect to the action of $\mathbf{g}_{\mathbf{1}}$ (resp. $\mathbf{g}_{\mathbf{2}}$ ). Let us denote by $a_{i, h}$ (resp. $b_{i, h}$ ) the number of points on $C_{1}$ (resp. $C_{2}$ ) whose stabilizer has order $h$ and such that the local action of $\mathbf{g}_{1}\left|\mathbf{g}_{1}\right| / h$ (resp. $\mathbf{g}_{2}{ }^{\left|\mathbf{g}_{2}\right| / h}$ ) is $\zeta_{h}^{i}$. Let $P \in C_{1}$ (resp. $Q \in C_{2}$ ) be a point with stabilizer of order $h$ (resp. $k$ ) where local action of $\mathbf{g}_{\mathbf{1}}$ (resp. $\mathbf{g}_{\mathbf{2}}$ ) is $\zeta_{h}^{i}$, $i \in\{1 \ldots h\}$ (resp. $\zeta_{k}^{j}, j \in\{1 \ldots k\}$ ). We assume that $h$ (resp. $k$ ) is a divisor of $\left|\mathbf{g}_{\mathbf{1}}\right|$ different from $\left|\mathbf{g}_{\mathbf{1}}\right|$. We will say that $P \times Q$ is a point of type $((i, h),(j, k))$ and clearly the number of these points is $a_{i, h} b_{j, k}$. The stabilizer of these points has order $d_{(h, k)}:=\operatorname{gcd}(h, k)$ and the orbit of $P \times Q$ w.r.t. $\mathbf{g}_{\mathbf{1}} \times \mathbf{g}_{\mathbf{2}}$ contains $\left|\mathbf{g}_{\mathbf{1}}\right| / d_{(h, k)}$ distinct points. In particular we observe that if $d_{(h, k)}=1$ the stabilizer of $P \times Q$ is empty and its orbit contains exactly $\left|\mathbf{g}_{\mathbf{1}}\right|$ points and if $i=j=\left|\mathbf{g}_{\mathbf{1}}\right|$, then the 
stabilizer of $P \times Q$ is $\mathbb{Z} /\left|\mathbf{g}_{\mathbf{1}}\right| \mathbb{Z}$ and the orbit of $P \times Q$ consists only of $P \times Q$. Let us now consider the quotient model $\left(C_{1} \times C_{2}\right) / G$ and the image $\overline{P \times Q}$ of the point $P \times Q$ for the quotient map: the point $\overline{P \times Q}$ is smooth if $d_{(h, k)}=1$; otherwise it is a singular points of type $\frac{1}{d_{(h, k)}}(1, q)$ where $q$ can be computed as in Proposition 5.3 of [3]. The images of the $a_{i, h} b_{j, k}$ points of type $((i, h),(j, k))$ under the quotient map consists of $a_{i, h} b_{j, k} d_{h, k} /\left|\mathbf{g}_{\mathbf{1}}\right|$ points.

Now we assume that $n=p$ is a prime number: then $h$ (resp. $k$ ) is necessarily 1 , hence $a_{i, h}=a_{i}$ (resp. $b_{j, k}=b_{j}$ ) with the notation of Section 2. The singular point $\overline{P \times Q} \in\left(C_{1} \times C_{2}\right) / G$ is of type $\frac{1}{p}\left(1, i j^{-1}\right)$, where $i j^{-1}$ is computed modulo $p$. Moreover, the orbit of every point with non trivial stabilizer consists only of one point and thus we have exactly $a_{i} b_{j}$ singular points on $\left(C_{1} \times C_{2}\right) / G$ which are of type $\frac{1}{p}\left(1, i j^{-1}\right)$.

Once one knows the number and the type of singularities of $\left(C_{1} \times C_{2}\right) / G$, one can easily compute $h^{1,1}(X)$, recalling that every singularity introduces a HJ-string, by Remark $3.3(2)$, and that

$$
\begin{aligned}
H^{1,1}\left(C_{1} \times C_{2}\right)^{G}= & \left(H^{1,1}\left(C_{1}\right) \otimes H^{0,0}\left(C_{2}\right)\right) \oplus\left(\oplus_{i}\left(H^{1,0}\left(C_{1}\right)_{\zeta_{n}^{i}} \otimes H^{0,1}\left(C_{2}\right)_{\zeta_{n}^{n-i}}\right)\right) \\
& \oplus\left(\oplus_{i}\left(H^{0,1}\left(C_{1}\right)_{\zeta_{n}^{i}} \otimes H^{1,0}\left(C_{2}\right)_{\zeta_{n}^{n-i}}\right)\right) \oplus\left(H^{0,0}\left(C_{1}\right) \otimes H^{1,1}\left(C_{2}\right)\right)
\end{aligned}
$$

In particular, since $H^{0,1}\left(C_{j}\right)_{\zeta_{n}^{i}} \simeq \overline{H^{1,0}\left(C_{j}\right)_{\zeta_{n}^{n-i}}}, j=1,2, h^{1,1}\left(C_{1} \times C_{2}\right)^{G}=$ $2\left(1+\sum_{i=1}^{n} \alpha_{i} \beta_{i}\right)$.

The following proposition recaps the results proved in this section.

Proposition 3.4. Let $C_{i}, i=1,2$ be a curve with an automorphism $\mathbf{g}_{\mathbf{i}}$ of order $n$. Let $\alpha_{l}:=\operatorname{dim}\left(H^{0,1}\left(C_{1}\right)_{\zeta_{n}^{l}}\right)$ and $\beta_{m}:=\operatorname{dim}\left(H^{0,1}\left(C_{2}\right)_{\zeta_{n}^{m}}\right)$. Let $a_{(i, h)}, b_{(j, k)}$ and $d_{(h, k)}$ as above. Note that $i$ and $j$ are invertible in $\mathbb{Z} / d_{(h, k)} \mathbb{Z}$ and we denote by $r_{i}$ and $s_{j}$ their inverses. Let $X$ be the minimal resolution of the quotient surface $\left(C_{1} \times C_{2}\right) /\left(\mathbf{g}_{1} \times \mathbf{g}_{2}\right)$. Then the Hodge numbers of $X$ are:

$$
\begin{aligned}
& h^{0,0}(X)=1, \quad h^{1,0}(X)=\alpha_{n}+\beta_{n}, \quad h^{2,0}(X)=\sum_{i=1}^{n} \alpha_{i} \beta_{n-1}, \\
& h^{1,1}(X)=2\left(1+\sum_{i=1}^{n} \alpha_{i} \beta_{i}\right)+\sum_{h, k} \sum_{i=1}^{n-1} \sum_{j=1}^{n-1}\left(a_{i, h} b_{j, k} d_{(h, k)} / n\right) k(i, j),
\end{aligned}
$$

where:

- we pose $k(i, j)=0$ if $d_{(h, k)}=1$;

- $k(i, j)$ is the number of curves introduced by a singularity of type $\frac{1}{d_{(h, k)}}(1, q)$, if $d_{(h, k)} \neq 1$;

- $q:=i s_{j} \in \mathbb{Z} / d_{(h, k)} \mathbb{Z}$ (or equivalently $\left.q:=j r_{i} \in \mathbb{Z} / d_{(h, k)} \mathbb{Z}\right)$.

Remark 3.5. We recall that $\alpha_{i}$ (resp. $\left.\beta_{i}\right)$ are uniquely determined by $a_{i}$ (resp. $\left.b_{i}\right)$ and viceversa, thus the Hodge numbers of $X$ depend only on the set of values $\left\{\alpha_{i}, \beta_{i}\right\}, i=1, \ldots, n$ (or equivalently on the set of values $\left\{a_{i}, b_{i}\right\}, i=1, \ldots, n$ ). 


\subsection{The minimal model $S$}

As observed in Remark 3.3(5), the surface $X$ is in general non minimal. Let us denote by $S$ the minimal model of $X$. Since $h^{i, 0}$ are birational invariant, the Hodge numbers $h^{0,0}, h^{1,0}, h^{2,0}$ of the product-quotient $X$ coincide with the ones of its minimal minimal model $S$. More complicated is the computation of $h^{1,1}(S)$.

In order to determine the minimal model $S$ of a product-quotient $X$, we have to find all the $(-1)$-curves on $X$. In the cases we will treat the $(-1)$-curves are central components of reducible fibres of one or both isotrivial fibrations of $X$. Then, after contractions of these, the $(-1)$-curves could be images of some divisors in the HJ-strings. Note that this is in general not true, see e.g., [5]. A quick method to calculate the self intersection of the central components is given in [27]. We shall recall it.

Definition 3.6. We say that a reducible fibre $F_{1}$ of $\pi_{2}: X \rightarrow C_{2} / G$ is of type $\left(q_{1} / n_{1}, \ldots, q_{r} / n_{r}\right)$ if it contains exactly $r H J$-strings $\widetilde{E}_{1}, \ldots, \widetilde{E}_{r}$, where each $\widetilde{E}_{i}$ is of type $\frac{1}{n_{i}}\left(1, q_{i}\right)$. The same definition holds for a reducible fibre $F_{2}$ of $\pi_{1}: S \rightarrow C_{1} / G$.

Proposition 3.7 (Proposition 2.8 in [27]). Let $F_{1}$ be of type $\left(q_{1} / n_{1}, \ldots, q_{r} / n_{r}\right)$, and let $Y_{1}$ be its central component. Then

$$
\left(Y_{1}\right)^{2}=-\sum_{i=1}^{r} \frac{q_{i}}{n_{i}} .
$$

If $F_{2}$ is of type $\left(q_{1}^{\prime} / n_{1}, \ldots, q_{r}^{\prime} / n_{r}\right)$, then $\left(Y_{2}\right)^{2}=-\sum_{i=1}^{r} q_{i}^{\prime} / n_{i}$.

In the following example we construct two surfaces, $S_{1}$ and $S_{2}$, both of them are the minimal model of quotients of $D_{3} \times D_{3}$ by a diagonal action of $\mathbb{Z} / 3 \mathbb{Z}$ but the action of this group is different in the two cases. As a consequence the minimal resolution of one quotient has an infinite number of $(-1)$-curves, the minimal resolution of the other has no $(-1)$-curves and the minimal models of these two resolutions are totally different: one of them is a rational surface, one is a K3 surface.

Example 3.8. Let us consider the product of two elliptic curves $D_{3} \times D_{3}$ and its automorphisms $\delta_{3} \times \delta_{3}^{1}$ and $\delta_{3} \times \delta_{3}^{2}$. Denote by $X_{i}$ the minimal resolution of $\left(D_{3} \times D_{3}\right) /\left(\delta_{3} \times \delta_{3}^{i}\right)$ and by $S_{i}$ its minimal model. Recall that $\delta_{3}^{i}$ acts on $H^{0,1}\left(D_{3}\right)$ as the multiplication by $\zeta_{3}^{i}$. We obtain $h^{0,0}\left(X_{i}\right)=h^{0,0}\left(S_{i}\right)=1, h^{1,0}\left(X_{i}\right)=h^{1,0}\left(S_{i}\right)=$ 0 for $i=1,2 ; h^{2,0}\left(X_{1}\right)=h^{2,0}\left(S_{1}\right)=0$ and $h^{2,0}\left(X_{2}\right)=h^{2,0}\left(S_{2}\right)=1$.

Now we compute $h^{1,1}\left(X_{i}\right)$ : on $\left(D_{3} \times D_{3}\right) /\left(\delta_{3} \times \delta_{3}\right)$ there are 9 singularities of type $\frac{1}{3}(1,1)$ and on $\left(D_{3} \times D_{3}\right) /\left(\delta_{3} \times \delta_{3}^{2}\right)$ there are 9 singularities of type $\frac{1}{3}(1,2)$. The resolution of a point of type $\frac{1}{n}(1,1)$ introduces 1 curve of self intersection $-n$, and thus $h^{1,1}\left(X_{1}\right)=9+\operatorname{dim}\left(H^{1,1}\left(D_{3} \times D_{3}\right)^{\delta_{3} \times \delta_{3}}\right)=13$. The desingularization of singularities of type $\frac{1}{n}(1, n-1)$ introduces $n-1$ rational curves with self intersection -2 , whose dual diagram is $A_{n-1}$, and thus $h^{1,1}\left(X_{2}\right)=2 \cdot 9+\operatorname{dim}\left(h^{1,1}\left(D_{3} \times D_{3}\right)^{\delta_{3} \times \delta_{3}^{2}}\right)=20$.

With the same method of the Section 7 we obtain that an equation for $\left(D_{3} \times\right.$ $\left.D_{3}\right) /\left(\delta_{3} \times \delta_{3}^{i}\right)$ is given by $y^{2}=x^{3}+\left(v^{2}-1\right)^{2 i}$, which is the equation of an elliptic 
fibration over $\mathbb{P}_{[v]}^{1}$. If $i=1$, this elliptic fibration is a rational elliptic fibration (this depend on the degree of $\left.\left(v^{2}-1\right)^{2 i}\right)$. By the standard theory of elliptic fibration (cf. [25]), it has 3 reducible fibers (over $1,-1$ and $\infty$ ) of type $I V$ (coming from the contraction of the central components of the reducible fibers on $X_{1}$ ). The rank of the Mordell-Weil group of this elliptic fibration is $2(=10-2-6$, by Corollary VII. 2.4 in [25], with $\rho=10$ and $\sum_{c \in \Delta} r_{c}=6$ ). This implies that there are infinite sections of this elliptic fibration, and thus an infinite number of $(-1)$-curves. Since the minimal elliptic fibration is obtained by contracting 3 $(-1)$-curves on $X_{1}$, we obtain that $X_{1}$ contains an infinite number of $(-1)$-curve. Since $X_{1}$ is birational to $\mathbb{P}^{2}$, there exists a minimal model of $X_{1}$ which is $S_{1}:=\mathbb{P}^{2}$ and $h^{1,1}\left(S_{1}\right)=1$.

The elliptic fibration $y^{2}=x^{3}+\left(v_{2}^{2}-1\right)^{4}$ has a K3 surface as minimal model. The reducible fibers of this elliptic fibration are 3 fibers of type $I V^{*}$ each of them consists of the central component (which is a (-2)-curve, by Proposition 3.7) and of three copy of $A_{2}$. So $X_{2}$ coincides with $S_{2}$ and $h^{1,1}\left(S_{2}\right)=20$. This K3 surface was constructed in [34].

\subsection{Automorphisms of $S$ and quotient surfaces}

By construction the surface $\left(C_{1} \times C_{2}\right) /\left(\mathbf{g}_{1} \times \mathbf{g}_{2}\right)$ admits an automorphism of order $n$ induced by $\mathrm{id} \times \mathrm{g}_{2} \in \operatorname{Aut}\left(\mathrm{C}_{1} \times \mathrm{C}_{2}\right)$ (or equivalently by $\mathbf{g}_{\mathbf{1}} \times \mathrm{id} \in \operatorname{Aut}\left(\mathrm{C}_{1} \times \mathrm{C}_{2}\right)$ ). This automorphism lifts to an automorphism of $X$ and of $S$. Thus, one can consider the quotient $\left(\left(C_{1} \times C_{2}\right) /\left(\mathbf{g}_{\mathbf{1}} \times \mathbf{g}_{\mathbf{2}}\right)\right) /\left(\mathrm{id} \times \mathbf{g}_{\mathbf{2}}\right)$. Since $\left\langle\mathbf{g}_{\mathbf{1}} \times \mathbf{g}_{\mathbf{2}}, \mathrm{id} \times \mathbf{g}_{\mathbf{2}}\right\rangle=\left\langle\mathbf{g}_{\mathbf{1}} \times\right.$ id, id $\left.\times \mathbf{g}_{\mathbf{2}}\right\rangle$, we have the following commutative diagram:

$$
\begin{array}{ccc}
\left.C_{1} \times C_{2}\right) / \mathbf{g}_{1} \times \mathbf{g}_{2} & \swarrow_{n: 1} \times C_{2} & \searrow^{n: 1} \\
& \searrow^{n: 1} & C_{1} / \mathbf{g}_{1} \times C_{2} / \mathbf{g}_{2} .
\end{array}
$$

This diagram lifts to the minimal resolution of all the surfaces we are considering, and so one obtains that the surface $X$ has a generically $n^{2}: 1$ map to $C_{1} / \mathbf{g}_{1} \times$ $C_{2} / \mathrm{g}_{2}$, and in particular to $\mathbb{P}^{1} \times \mathbb{P}^{1}$, if we assume $C_{i} / \mathrm{g}_{\mathbf{i}} \simeq \mathbb{P}^{1}$. Moreover, the map $X \rightarrow \mathbb{P}^{1} \times \mathbb{P}^{1}$ induces a rational $n^{2}: 1 \operatorname{map} S \rightarrow \mathbb{P}^{1} \times \mathbb{P}^{1}$.

We can explicitly describe the action of id $\times \mathbf{g}_{\mathbf{2}}$ on the cohomology of $X$ (we keep the assumption $\left.C_{i} / \mathbf{g}_{\mathbf{i}} \simeq \mathbb{P}^{1}\right)$ : id $\times \mathbf{g}_{\mathbf{2}}$ acts as the identity on the spaces $H^{0,0}(X)$ and $H^{1,0}(X)$. The invariant subspace of $H^{2,0}(X)$ under id $\times \mathbf{g}_{2}$ is the image of the space $H^{1,0}\left(C_{1}\right)^{\mathbf{g}_{1}} \otimes H^{1,0}\left(C_{2}\right)^{\mathbf{g}_{2}}$ and thus has dimension $\alpha_{n} \beta_{n}=0$. As we saw in Section 3.2, the space $H^{1,1}(X)$ splits into two parts: the image of $H^{1,1}\left(C_{1} \times\right.$ $\left.C_{2}\right)^{\mathbf{g}_{1} \times \mathbf{g}_{2}}$ and a direct summand, say $R$, which comes from the resolution of the singularities of $\left(C_{1} \times C_{2}\right) /\left(\mathbf{g}_{1} \times \mathbf{g}_{2}\right)$. Hence, $H^{1,1}(X)^{\mathrm{id} \times \mathbf{g}_{2}}$ splits into the direct sum of the image of $\left(H^{0,0}\left(C_{1}\right) \otimes H^{1,1}\left(C_{2}\right)\right) \oplus\left(H^{1,0}\left(C_{1}\right)^{\mathbf{g}_{1}} \otimes H^{0,1}\left(C_{2}\right)^{\mathbf{g}_{2}}\right) \oplus\left(H^{0,1}\left(C_{1}\right)^{\mathbf{g}_{\mathbf{1}}} \otimes\right.$ $\left.H^{1,0}\left(C_{2}\right)^{\mathbf{g}_{2}}\right) \oplus\left(H^{1,1}\left(C_{1}\right) \otimes H^{0,0}\left(C_{2}\right)\right)$ and $R^{\mathrm{id} \times \mathbf{g}_{2}}$. The dimension of the first term is $2+2 \alpha_{n} \beta_{n}=2$. We note that, if every point in the branch locus of $C_{i}, i=1,2$ is of total ramification, then the action of id $\times \mathrm{g}_{2}$ is the identity on $R$, and one finds $H^{1,1}(X)^{\mathrm{id} \times \mathbf{g}_{2}}=\left(H^{0,0}\left(C_{1}\right) \otimes H^{1,1}\left(C_{2}\right)\right) \oplus\left(H^{1,1}\left(C_{1}\right) \otimes H^{0,0}\left(C_{2}\right)\right) \oplus(R \otimes \mathbb{C})$. 


\section{K3 surfaces}

This section is devoted to the construction of K3 surfaces $S$, which are minimal model of product-quotient surfaces.

We recall that by definition a $K 3$ surface $S$ has $h^{1,0}(S)=0$ and trivial canonical bundle. The Hodge numbers of $S$ are uniquely determined by these properties and are $h^{0,0}(S)=h^{2,0}(S)=1, h^{1,0}(S)=0, h^{1,1}(S)=20$.

\subsection{Product-quotient surfaces with $p_{g}=1$ and $q=0$}

Let $S$ be the minimal model of a product-quotient $X$ with quotient model $\left(C_{1} \times\right.$ $\left.C_{2}\right) /\left(\mathbf{g}_{\mathbf{1}} \times \mathbf{g}_{\mathbf{2}}\right)$. If $S$ is a K3 surface, then $q(S)=h^{1,0}(S)=0$ and $p_{g}(S)=h^{2,0}(S)=1$. Since $h^{i, 0}$ are birational invariants, $h^{1,0}(X)=0, h^{2,0}(X)=1$. Therefore, by Proposition 3.4, $\alpha_{n}+\beta_{n}=0$ and $\sum_{i=1}^{n} \alpha_{i} \beta_{n-i}=1$. In particular,

$$
\begin{aligned}
& \alpha_{n}=\beta_{n}=0 \text { and there exists } i \in\{1, \ldots, n\} \text { such that } \\
& \qquad \alpha_{i}=\beta_{n-i}=1, \quad \alpha_{j} \beta_{n-j}=0 \text { if } j \neq i .
\end{aligned}
$$

Condition (4.1) is divided in two: a condition on each factor of the product, namely the action of $\mathbf{g}_{\mathbf{i}}$ on $H^{1,0}\left(C_{i}\right)$; and a condition on the whole product, namely the action of $\mathbf{g}_{\mathbf{1}} \times \mathbf{g}_{\mathbf{2}}$ on $H^{2,0}\left(C_{1} \times C_{2}\right)$.

Now we shall give a procedure to construct product-quotient surfaces with $p_{g}=$ 1 and $q=0$. Since, in general, this algorithm requires excessively long calculations, many of them are performed using the Magma program. While describing the procedure we also indicate which part of the program does what.

First we search for pairs $\left(C_{1}, \mathbf{g}_{1}\right)$ which satisfy the following two conditions: $\alpha_{n}=0$, or equivalently $C_{i} / \mathbf{g}_{\mathbf{i}} \simeq \mathbb{P}^{1}$; and there exists an index $0<j<n$ such that $\alpha_{j}=1$. The first condition is obtained by giving spherical systems of generators for $G$ (see Theorem 2.1). The second one is obtained exploiting the ChevalleyWeil formula and calculating the rotation angles as in Proposition 2.3. Analogous conditions must hold for a second pair $\left(C_{2}, \mathbf{g}_{2}\right)$.

Second we couple the curves by requiring that there exists an $i \in\{1 \ldots n\}$ such that $\alpha_{i}=\beta_{n-i}=1$ and $\alpha_{j} \beta_{n-j}=0$ if $j \neq i$.

Let us assume that $n=p$ a prime. The curves $C_{i}$ admitting an automorphism $\mathbf{g}_{\mathbf{i}}$ of order $p$ such that $C_{i} / \mathbf{g}_{\mathbf{i}} \simeq \mathbb{P}^{1}$ and $\alpha_{j}=1$ for some $j \in\{1, \ldots, p-1\}$, have genus at most $(p-1)^{2}$, by Corollary 2.4. The number of the ramification points of the cover $C_{i} \rightarrow C_{i} / \mathbf{g}_{\mathbf{i}}$ is at most $2 p$. This implies that the number of curves with these properties is finite. These curves are classified by the Magma program, using the function Surfacesp. The function calculates all the partitions of all the numbers from 3 up to $2 p$, giving all the admissible ramification data $\left(a_{1}, \ldots, a_{n-1}\right)$ of the coverings $C_{i} \rightarrow C_{i} / g_{i} \simeq \mathbb{P}^{1}$. Afterwards it evaluates the $\alpha_{j}$, and lists only the ones with $\alpha_{j}=1$ for at least one $j \in\{1, \ldots, p-1\}$.

Remark 4.1. We observe that for every prime $p$ there exists at least one curve with the required properties, the one with $a_{p-1}=2$ and $a_{(p+1) / 2}=1$ (cf. Example 2.5). 
The condition $\alpha_{j} \beta_{n-j}=0$ if $j \neq i$ implies that the list $\left(\alpha_{1}, \ldots, \alpha_{p-1}, \beta_{1}, \ldots \beta_{p-1}\right)$ contains at least $p-2$ zeros. This condition is verified in the function MaybeSur 1 of the Magma program. Then, the function TheSur tests if a surface given by MaybeSur1 has also $p_{g}=1$.

Proposition 4.2. There exists a finite number of families of surfaces $S$ which are the minimal model of $\left(C_{1} \times C_{2}\right) /\left(\mathbf{g}_{1} \times \mathbf{g}_{2}\right)$, with $\left|\left(\mathbf{g}_{\mathbf{1}} \times \mathbf{g}_{\mathbf{2}}\right)\right|=p$, and $p_{g}(S)=1$ and $q(S)=0$.

Proof. For a given $p$, the number of surfaces $S$ is finite, since the numbers of pairs $\left(C_{i}, \mathbf{g}_{\mathbf{i}}\right)$ is so (cf. Corollary 2.4$)$.

The automorphism id $\times \mathbf{g}_{2}$ induces an automorphism on $S$ which acts non trivially on $H^{2,0}(S)$, see Section 3.4. In order to give a bound for $p$ we prove that there exist no a surface $Z$ with $p_{g}(Z)=1, q(Z)=0$ and an automorphism of order $p>19$ acting non trivially on $H^{2,0}(Z)$.

Let $Z$ be a minimal surface with $h^{2,0}(Z)=1$ which admits an automorphism $\sigma$ of order $p$ and let $H^{2}(Z, \mathbb{C})_{\zeta_{p}^{i}}$ be the eigenspace of the eigenvalue $\zeta_{p}^{i}$ for the action of $\sigma, i=0, \ldots, p-1$. The dimension of $H^{2}(Z, \mathbb{C})_{\zeta_{p}^{i}}$ does not depend on $i$ if $i \neq 0$. Thus, if there exists $i \neq 0, i \in\{1, \ldots, p-1\}$ such that $\operatorname{dim}\left(H^{2}(Z, \mathbb{C})_{\zeta_{p}^{i}}\right) \geq 1$, then $b_{2}(Z) \geq p-1$.

Since $h^{2,0}(Z)=1, K_{Z}^{2} \geq 0$. By $h^{1,0}(Z)=0$ follows that $\chi(Z)=2$ and that $e(Z) \leq 24$ by Noether equality. So $b_{2}(Z) \leq 22$.

Since there exists no a surface $Z$ with $p_{g}(Z)=1 q(Z)=0$ and $b_{2}(Z)>22$, there exists no a surfaces $Z$ with $p_{g}(Z)=1, q(Z)=0$ and an automorphism of order $p>19$ acting non trivially on $H^{2,0}(Z)$.

Remark 4.3. Since a minimal surface with $p_{g}=1, q=0$ can not admit an automorphism of order $p>19$, there exist no pairs $\left(C_{1} \times C_{2}, \mathbf{g}_{1} \times \mathbf{g}_{2}\right)$ such that $\left|\mathbf{g}_{1} \times \mathbf{g}_{2}\right|=p>19, \operatorname{dim}\left(H^{1}\left(C_{1} \times C_{2}\right)^{\mathbf{g}_{1} \times \mathbf{g}_{2}}\right)=0$ and $\operatorname{dim}\left(H^{2,0}\left(C_{1} \times C_{2}\right)^{\mathbf{g}_{1} \times \mathbf{g}_{2}}\right)=1$.

Lemma 4.4. Let $X$ be the minimal resolution of $\left(C_{1} \times C_{2}\right) / G$. Let $F_{i}$ be the fiber of $\pi_{j}: X \rightarrow C_{j} / G,\{i, j\}=\{1,2\}$. We recall $F_{i} \simeq C_{i}$. If $q(X)=0$, then the linear systems $\left|F_{i}\right|$ on $X, i=1,2$ are complete and of dimension 1 .

Proof. Since $F_{i}^{2}=0$, we have the short exact sequence

$$
0 \rightarrow \mathcal{O}\left(K_{X}-F_{i}\right) \rightarrow \mathcal{O}\left(K_{X}\right) \rightarrow \mathcal{O}_{F_{i}}\left(K_{F_{i}}\right) \rightarrow 0 .
$$

This induces the long exact sequence in cohomology

$H^{1}\left(X, \mathcal{O}\left(K_{X}\right)\right) \rightarrow H^{1}\left(F_{i}, \mathcal{O}\left(K_{F_{i}}\right)\right) \rightarrow H^{2}\left(X, \mathcal{O}\left(K_{X}-F_{i}\right)\right) \rightarrow H^{2}\left(X, \mathcal{O}\left(K_{X}\right)\right) \rightarrow 0$.

By Serre duality, $\operatorname{dim}\left(H^{1}\left(X, \mathcal{O}\left(K_{X}\right)\right)\right)=q(X)=0$ and $H^{2}\left(X, \mathcal{O}\left(K_{X}-F_{i}\right)\right)=$ $H^{0}\left(X, \mathcal{O}\left(F_{i}\right)\right)$. Therefore,

$$
\begin{aligned}
\operatorname{dim}\left(H^{0}\left(X, \mathcal{O}\left(F_{i}\right)\right)\right) & =\operatorname{dim}\left(H^{1}\left(F_{i}, \mathcal{O}\left(K_{F_{i}}\right)\right)\right)+\operatorname{dim}\left(H^{2}\left(X, \mathcal{O}\left(K_{X}\right)\right)\right) \\
& =\operatorname{dim}\left(H^{0}\left(F_{i}, \mathcal{O}\right)\right)+1=2 .
\end{aligned}
$$


Proposition 4.5. Let $X$ be the minimal resolution of $\left(C_{1} \times C_{2}\right) / G$. If $q(X)=0$, the dimension of the family of product-quotient surfaces $X$ is $r_{1}+r_{2}-6$, where $C_{i} \rightarrow C_{i} / G$ is ramified in $r_{i}$ points. Moreover this family is a quasi-projective irreducible variety.

Proof. By Proposition 2.1, the number $\eta$ of parameters of the family of productquotient surfaces is less than or equal to $r_{1}+r_{2}-6$. For simplicity we assume $r_{2}=3$. If $\eta<r_{1}-3$, then there exists a positive dimensional family of curves isomorphic to $C_{1}$ which induces a family of isotrivial fibrations on $X$, whose fibers are isomorphic to $C_{1}$. Since $q(X)=0, \operatorname{Pic}(X)=N S(X) /$ Tors $\simeq \mathbb{Z}^{N}$ for a certain positive integer $N$. By Lemma 4.4, the linear system $\left|F_{1}\right|$ is complete and of dimension 1. Since $\operatorname{Pic}(X)$ is discrete there is no positive dimensional family of such linear systems. Therefore $\eta=r_{1}-3$. The family is quasi-projective and irreducible by Theorem 5.4 in [6].

As stated in Proposition 4.2, we are looking for a finite number of surfaces. These are given by the program Surfacesp. However the number of permutations of the ramification points increases too rapidly with the growth of $p$ for a computation in a short time. Since our aim was the construction of K3 surfaces, and we know the dimension of the families we are searching for, we wrote another program with a fixed number of ramification points, and hence with fixed dimension of the family. The program t1t2PtsSurfaces, given a cyclic group $G$ of order $p$ or $2 p$ and the numbers $t i, i=1,2$, of ramification points of $C_{i} \rightarrow C_{1} / G \simeq \mathbb{P}^{1}$, returns a list of product-quotient surfaces $T$ with $p_{g}(T)=1$ and $q(T)=0$, as well as the singularities of $T$.

Remark 4.6. If $\left|\mathbf{g}_{\mathbf{1}}\right|=2 p$, then by Remark 2.4 there exists a finite list of curves with at least one $\alpha_{j}=1$ and $\zeta_{2 p}^{j}$ is a primitive $2 p$-root of unity. If the action of $\mathbf{g}_{1}$ on $C_{1}$ is of this type, the same must be true for the action of $\mathbf{g}_{2}$ on $C_{2}$, thus we have a finite list for $\left(C_{1} \times C_{2}\right) /\left(\mathbf{g}_{1} \times \mathbf{g}_{2}\right)$. Hence, we obtain a complete classification of such surfaces as in Proposition 4.2. Otherwise, if we assume that the action of $\mathbf{g}_{1}$ on $C_{1}$ is such that $\alpha_{j}=1$ and $\zeta_{2 p}^{j}$ is a either a primitive $p$-root of unity or $(-1)$, then the same must be true for the action of $\mathbf{g}_{2}$ on $C_{2}$. In this case we can not construct a complete list of the curves $C_{1}$ and $C_{2}$, since we have no an upper bound for their genera, and so for the number of ramification points of the map $f_{i}: C_{i} \rightarrow C_{i} / \mathbf{g}_{\mathbf{i}} \simeq \mathbb{P}^{1}$. Anyway, if we fix the maximal number $n$ of ramification points for $f_{i}$, then we obtain a finite list of curves $C_{i}$ and thus a finite number of surfaces $\left(C_{1} \times C_{2}\right) /\left(\mathbf{g}_{1} \times \mathbf{g}_{2}\right)$ as in Proposition 4.2. Our aim is to construct K3 surfaces, so the bound on $n$ depends on the dimension of the moduli space of K3 surfaces. More precisely, the moduli space of projective K3 surfaces has dimension 19 , so the sum of the ramification points of $f_{1}$ and $f_{2}$ can not be grater then 25 .

\subsection{K3 surfaces}

Let $S$ be the minimal model of $\left(C_{1} \times C_{2}\right) /\left(\mathbf{g}_{1} \times \mathbf{g}_{2}\right)$. If $S$ is a K3 surface, then by definition $p_{g}(S)=1$ and $q(S)=0$. Therefore the K3 surfaces obtained as minimal 
model of $\left(C_{1} \times C_{2}\right) /\left(\mathbf{g}_{1} \times \mathbf{g}_{2}\right)$ are among the ones listed in Section 4.1. In order to prove that $S$ is a K3 surface one has to verify that the canonical bundle is trivial.

Lemma 4.7. Let $Z$ be a surface obtained contracting $-K_{X}^{2}(-1)$-curves on $X$. We recall that $p_{g}(Z)=1$ and $q(Z)=0$. Let $F_{1}$ be the class of the fiber of the fibration $\pi_{2}: X \rightarrow C_{2} /\left\langle\mathbf{g}_{2}\right\rangle$ and $F_{2}$ be the class of the fiber of the fibration $\pi_{1}: X \rightarrow$ $C_{1} /\left\langle\mathbf{g}_{1}\right\rangle$. Let $E$ be the sum of all the exceptional divisors of the blow up $X \rightarrow Z$. If $\left(K_{X}-E\right) F_{1}=0$ and $\left(K_{X}-E\right) F_{2}=0$, then $K_{Z}$ is trivial. In this case $Z$ is minimal.

Proof. We shall denote by $P_{k}$ the singular points of $\left(C_{1} \times C_{2}\right) /\left(\mathbf{g}_{1} \times \mathbf{g}_{2}\right)$, and by $A_{j, k}$ the $j$-th irreducible component of the HJ-string of the blow-up $\pi: X \rightarrow$ $\left(C_{1} \times C_{2}\right) /\left(\mathbf{g}_{1} \times \mathbf{g}_{2}\right)$ over $P_{k}$.

Let $D$ be an effective divisor on $X$, then $D=\lambda_{1} F_{1}+\lambda_{2} F_{2}+\lambda_{3} B+\sum_{j, k} \lambda_{j, k} A_{j, k}$ with $\lambda_{i}, \lambda_{j, k} \geq 0$ and $B F_{1}>0, B F_{2}>0$. If $D F_{1}=0$ and $D F_{2}=0$, then $\lambda_{1}=\lambda_{2}=\lambda_{3}=0$. For every $k, \cup_{j} A_{j, k}$ is a HJ-string, hence $D A_{j, k}=0$ for every $j$ and $k$. Therefore $D F_{1}=D F_{2}=0$ give a homogeneous linear system in $\lambda_{j, k}$. The corresponding matrix is a diagonal block matrix and each block is invertible, being associated to the resolution of the quotient singularity $P_{k}$. Thus $D=0$.

The divisor $K_{X}-E$ is an effective divisor being the pullback of the canonical divisor of $Z$, which has $p_{g}(Z)=1$. Applying the previous result to $D=K_{X}-E$ we obtain $K_{X}-E=0$ hence $K_{Z}$ is trivial.

Necessary conditions to obtain a K3 surface $S$ as minimal model of the minimal resolution $X$ of the quotient $\left(C_{1} \times C_{2}\right) /\left(\mathbf{g}_{1} \times \mathbf{g}_{2}\right)$ are the following:

1. $h^{i, 0}(X)=1$, for $i=0,2$;

2. $h^{1,0}(X)=0$;

3. there are exactly $-K_{X}^{2}(-1)$-curves on $X$.

Thus, in order to classify the $\mathrm{K} 3$ surfaces $S$, we list the surfaces $X$ satisfying the conditions (1), (2). If $\left|\mathbf{g}_{\mathbf{1}} \times \mathbf{g}_{\mathbf{2}}\right|=p$ is prime number, as already said, this is done by the program Surfacesp.

Next we consider the $(-1)$-curves which are either central components $Y$ of reducible fibers (we calculate $Y^{2}$ using Proposition 3.7) or appear as image of curves in HJ-string after some contractions. In this way we find $-K_{X}^{2}(-1)$-curves. After the contraction of all these curves we always obtain a surface which satisfies the condition of Lemma 4.7 and so a surface $Z$ with trivial canonical bundle. This implies that there are no other $(-1)$-curves on $Z$, which is thus the minimal model $S$ of $X$ and in particular $S$ is a K3 surface.

\subsection{Non-symplectic automorphisms}

We saw in Section 3.4 that every surface $S$ which is the minimal model of a productquotient with $\mathbf{g}_{\mathbf{1}} \times \mathbf{g}_{\mathbf{2}}$ admits an automorphism induced by $\mathrm{id} \times \mathbf{g}_{\mathbf{2}}$ which acts non trivially on $H^{2,0}(S)$. This means that if $S$ is a K3 surface, the automorphism induced on $S$ by id $\times \mathbf{g}_{2}$ is a purely non-symplectic automorphism on $S$. Thus the surface $S$ admits a non-symplectic automorphism of prime order. 
Definition 4.8. Let $W$ be a K3 surface. Let $\omega_{W}$ be a generator of $H^{2,0}(W)$. An automorphism $\mathbf{g} \in \operatorname{Aut}(W)$ of order $n$ is called symplectic if $\mathbf{g}\left(\omega_{W}\right)=\omega_{W}$, and purely non-symplectic if $\mathbf{g}\left(\omega_{W}\right)=\zeta_{n}^{i} \omega_{W}$ and $\zeta_{n}^{i}$ is a primitive $n$-root of unity.

We observe that an automorphism of prime order $p$ which is non-symplectic is purely non-symplectic. This type of automorphism are classified [2]. In this section we summarize the main results on non-symplectic automorphisms of prime order, which will be considered in the following.

For every prime number $2 \leq p \leq 19$ there exists a K3 surface $W$ admitting a non-symplectic automorphism $\mathrm{g}$ of order $p$. Let us assume $3 \leq p \leq 19$. The fixed locus Fix $\mathbf{g}(W)=\{w \in W$ such that $\mathbf{g}(w)=w\}$ consists of the disjoint union of $n$ isolated points and $k+1$ curves. At most one of the fixed curves has a positive genus (see Theorems 4.1, 5.3, 6.3, 7.3, and 8.4 in [2]), and we denote by $g(C)$ the genus of the curve with highest genus. Hence, the fixed locus consists of $n$ isolated points, $k$ rational curves and another curve $C$ with a possibly positive genus. For each prime number $3 \leq p \leq 19$ such that $|\mathbf{g}|=p$, there exists a finite number of possibilities for the fixed locus $\operatorname{Fix}_{\mathbf{g}}(W)$, and the fixed locus is uniquely determined by the data $n, g(C), k+1$. So $(p, n, g(C), k+1)$ is an invariant which determines the fixed locus. For each prime $p=|\mathbf{g}|$, the admissible choices for $(n, g(C), k+1)$ are listed in Theorems 4.1, 5.3, 6.3, 7.3, and 8.4 of [2], where it is also proved that there exists a K3 surface with a non-symplectic automorphism of order $p$ with fixed locus determined by $(n, g(C), k+1)$ for every admissible choice of the invariants.

More precisely, the invariants $(p, n, g(C), k+1)$ determine uniquely the two lattices $S_{(n, g(C), k+1)}^{p}:=H^{2}(W, \mathbb{Z})^{\mathbf{g}}$ and $T_{(n, g(C), k+1)}^{p}:=\left(H^{2}(W, \mathbb{Z})^{\mathbf{g}}\right)^{\perp}$ and a $\mathrm{K} 3$ surface admits a non-symplectic automorphism of order $p$ with fixed locus determined by $(n, g(C), k+1)$ only if $S_{(n, g(C), k+1)}^{p}$ is primitively embedded in its Néron-Severi group. This allows one to describe the family of K3 surfaces with a non-symplectic automorphism of order $p$ and a prescribed fixed locus in terms of the period map of K3 surfaces. We will denote by $\mathcal{M}_{(n, g(C), k+1)}^{p}$ the family of K3 surfaces admitting a non-symplectic automorphism of order $p$ with fixed locus determined by $(n, g(C), k+1)$. It has one connected component of dimension $\left(\operatorname{rk}\left(T_{(n, g(C), k+1)}^{p}\right) /(p-1)\right)-1$. To give a more precise description of the moduli space of the K3 surfaces that admit a non-symplectic automorphism of order $p$ and a prescribed fixed locus, we consider the action of $\mathbf{g}$ on $T_{(n, g(C), k+1)}^{p} \otimes \mathbb{C}$, which does not depend on the K3 surface considered. By definition $\mathrm{g}$ has no eigenvalue 1 on $T_{(n, g(C), k+1)}^{p} \otimes \mathbb{C}$ and the decomposition in eigenspaces consists of $p-1$ equidimensional eigenspaces (of the eigenvalues $\zeta_{p}^{i}, i=1, \ldots p-1$ ). Let $\left(T_{(n, g(C), k+1)}^{p} \otimes \mathbb{C}\right)_{\zeta_{p}}$ be the unique eigenspace such that $\left(T_{(n, g(C), k+1)}^{p} \otimes \mathbb{C}\right)_{\zeta_{p}}^{2,0} \neq 0$. Set $\mathcal{B}:=\left\{z \in \mathbb{P}\left(\left(T_{(n, g(C), k+1)}^{p} \otimes \mathbb{C}\right)_{\zeta_{p}}\right)\right.$ such that $\left.(z, z)=0, \quad(z, \bar{z})>0\right\}$. The space $\mathcal{B}$ is a ball of dimension $\left(\operatorname{rk}\left(T_{(n, g(C), k+1)}^{p}\right) /(p-1)\right)-1$. Let $\Gamma:=\{\gamma \in$ $\mathrm{O}\left(T_{(n, g(C), k+1)}^{p} \otimes \mathbb{C}\right)$ such that $\left.\gamma \mathbf{g}=\mathbf{g} \gamma\right\}$. There is a birational map between the space of the K3 surfaces admitting a non-symplectic automorphism as required and $\mathcal{B} / \Gamma$ (see Section 11 of $[11]$ ). The family $\mathcal{M}_{(n, g(C), k+1)}^{p}$ is an irreducible quasiprojective variety (see Theorem 9.1 and Proposition 9.3 in [2]). 
For a fixed prime number $3 \leq p \leq 19$, there are some inclusions among the families $\mathcal{M}_{(n, g(C), k+1)}^{p}$; for example, $\mathcal{M}_{(9,0,6)}^{3} \subset \overline{\mathcal{M}_{(8,0,5)}^{3}}$. All these inclusions are described in [2], and the maximal components are determined in Theorem 9.5 of [2]:

if $p=3$, there are three maximal components: $\mathcal{M}_{(3,-, 0)}^{3}, \mathcal{M}_{(0,4,1)}^{3}, \mathcal{M}_{(0,5,2)}^{3}$ and for every admissible data $(n, g(C), k+1)$, we have $\mathcal{M}_{(n, g(C), 1)}^{3} \subset \overline{\mathcal{M}_{(0,4,1)}^{3}}$ and $\mathcal{M}_{(n, g(C), k+1)}^{3} \subset \overline{\mathcal{M}_{(0,5,2)}^{3}}$ if $k+1 \geq 2$. The dimension of each family is $m=9-n$;

if $p=5,7,11$, there are two maximal components: $\mathcal{M}_{(n,-, 0)}^{p}$, and $\mathcal{M}_{(n, g(C), 1)}^{p}$ and for every admissible data $(n, g(C), k+1)$, we have $\mathcal{M}_{(n, g(C), k+1)}^{p} \subset \overline{\mathcal{M}_{\left(n^{\prime}, g\left(C^{\prime}\right), 1\right)}^{p}}$ if $k+1 \neq 0$. The dimension of each family is $m=(13-n) /(p-2)$ if $p=5,7$ and $m=(11-n) /(p-2)$ if $p=11$;

if $p=13,17,19$, there is only one (rigid) K3 surface admitting a non-symplectic automorphism of order $p$. Thus there is one maximal component (in fact one component of dimension 0) which is $\mathcal{M}_{(9,0,1)}^{13}, \mathcal{M}_{(7,-, 0)}^{17}, \mathcal{M}_{(5,-, 0)}^{19}$ respectively.

The K3 surfaces we are constructing as product-quotients are members of the families of K3 surfaces admitting a non-symplectic automorphism of prime order. In the following sections we will construct K3 surfaces $S$ and we will determine the fixed locus of the non-symplectic automorphism induced by $\mathrm{id} \times \mathbf{g}_{2}$ (or by some of its powers), and thus we identify on which component of the family of K3 surfaces with a non-symplectic automorphisms they lie.

The following remark is used to determine the fixed locus.

Remark 4.9. Let us assume $\left|\mathbf{g}_{\mathbf{2}}\right|=p$ is a prime number. The automorphism $\mathbf{g}_{S}$ of $S$ induced by id $\times \mathrm{g}_{2}$ fixes the central components of all the reducible fibers of the fibration $\pi_{2}$. Similarly, it fixes the central components of all the reducible fibers of the fibration $\pi_{1}$ since id $\times \mathbf{g}_{\mathbf{2}} \equiv \mathbf{g}_{\mathbf{1}} \times$ id $\bmod \left(\mathbf{g}_{1} \times \mathbf{g}_{\mathbf{2}}\right)$. Moreover, it fixes all the singular points of the HJ-strings introduced resolving the singularities of $\left(C_{1} \times C_{2}\right) /\left(\mathbf{g}_{1} \times \mathbf{g}_{2}\right)$. It is possible that the automorphism fixes some disjoint components of certain HJ-strings which do not meet any other fixed curves.

\section{K3 surfaces which are minimal models of $\left(C_{1} \times C_{2}\right) /(\mathbb{Z} / p \mathbb{Z})$}

The aim of this section is to list and to describe the K3 surfaces obtained as minimal model of $\left(C_{1} \times C_{2}\right) /\left(\mathbf{g}_{1} \times \mathbf{g}_{2}\right)$ with $\left|\mathbf{g}_{1} \times \mathbf{g}_{2}\right|=p$.

Theorem 5.1. All the K3 surfaces which are minimal models of $\left(C_{1} \times D_{p}\right) /\left(\mathbf{g}_{1} \times\right.$ $\left.\delta_{p}\right),\left|\mathbf{g}_{\mathbf{1}} \times \mathbf{g}_{\mathbf{2}}\right|=p$ admit a non-symplectic automorphism of order $p$ whose fixed locus is one of those listed in Table 1.

Viceversa, let $S$ be a K3 surface, generic in the family of the K3 surfaces admitting a non-symplectic automorphism of odd prime order $p$ with fixed locus listed in column $(n, g, k+1)$ of Table 1 . Then $S$ is minimal the model of $\left(C_{1} \times C_{2}\right) /$ $\left(\mathbf{g}_{\mathbf{1}} \times \mathbf{g}_{\mathbf{2}}\right)$ with $\left|\mathbf{g}_{\mathbf{1}} \times \mathbf{g}_{\mathbf{2}}\right|=p$, and for each such surface $S$ we can choose $\left(C_{2}, \mathbf{g}_{\mathbf{2}}\right) \simeq$ $\left(D_{p}, \delta_{p}\right)$ and the non-symplectic automorphism on $S$ is always induced by $\mathrm{id} \times \delta_{\mathrm{p}}$. 
Moreover, the same holds true for every $K 3$ surface admitting a non-symplectic automorphism of odd prime order $p$ with fixed locus listed in the column $(n, g, k+1)$ of the Table 1 and with minimal Picard number.

\begin{tabular}{|c|c|c|c|c|c|c|c|}
\hline$p$ & $g\left(C_{1}\right)$ & $\left(\alpha_{1}, \ldots \alpha_{p-1}\right)$ & $\left(a_{1}, \ldots, a_{p-1}\right)$ & $\operatorname{Sing}\left(C_{1} \times D_{p} / \mathbf{g}_{1} \times \delta_{p}\right)$ & $K_{X}^{2}$ & $(n, g, k+1)$ & $m$ \\
\hline 3 & 4 & $(3,1)$ & $(0,6)$ & $\left(\frac{1}{3}\right)^{18}$ & -6 & $(6,0,3)$ & 3 \\
\hline 3 & 3 & $(2,1)$ & $(1,4)$ & $\left(\frac{1}{3}\right)^{12},\left(\frac{2}{3}\right)^{3}$ & -4 & $(7,0,4)$ & 2 \\
\hline 3 & 2 & $(1,1)$ & $(2,2)$ & $\left(\frac{1}{3}\right)^{6},\left(\frac{2}{3}\right)^{6}$ & -2 & $(8,0,5)$ & 1 \\
\hline 3 & 1 & $(0,1)$ & $(3,0)$ & $\left(\frac{2}{3}\right)^{9}$ & 0 & $(9,0,6)$ & 0 \\
\hline 5 & 6 & $(3,2,1,0)$ & $(0,0,0,5)$ & $\left(\frac{1}{5}\right)^{10},\left(\frac{3}{5}\right)^{5}$ & -12 & $(7,0,1)$ & 2 \\
\hline 5 & 4 & $(2,1,1,0)$ & $(0,1,0,3)$ & $\left(\frac{1}{5}\right)^{6},\left(\frac{2}{5}\right)^{5},\left(\frac{4}{5}\right)$ & -8 & $(10,0,2)$ & 1 \\
\hline 5 & 2 & $(1,0,1,0)$ & $(0,2,0,1)$ & $\left(\frac{1}{5}\right)^{2},\left(\frac{2}{5}\right)^{5},\left(\frac{4}{5}\right)^{2}$ & -4 & $(13,0,3)$ & 0 \\
\hline 7 & 6 & $(2,2,1,1,0,0)$ & $(0,0,0,0,1,3)$ & $\left(\frac{1}{7}\right)^{6},\left(\frac{3}{7}\right)^{3},\left(\frac{4}{7}\right)^{2},\left(\frac{5}{7}\right)$ & -14 & $(8,0,1)$ & 1 \\
\hline 7 & 3 & $(0,1,1,0,0,1)$ & $(0,0,0,2,1,0)$ & $\left(\frac{1}{7}\right)^{2},\left(\frac{2}{7}\right)^{2},\left(\frac{3}{7}\right)^{5}$ & -7 & $(13,0,2)$ & 0 \\
\hline 11 & 5 & $(1,1,0,1,1$, & $(0,0,1,0,0$, & $\left(\frac{1}{11}\right)^{2},\left(\frac{2}{11}\right),\left(\frac{3}{11}\right)^{2}$, & -13 & $(11,0,1)$ & 0 \\
& & $0,0,1,0,0)$ & $0,1,0,0,1)$ & $\left(\frac{4}{11}\right),\left(\frac{5}{11}\right),\left(\frac{7}{11}\right)^{2}$ & & & \\
\hline 13 & 6 & $(1,1,1,0,1,1$, & $(0,0,0,1,0,0$, & $\left(\frac{1}{13}\right)^{2},\left(\frac{2}{13}\right),\left(\frac{3}{13}\right)^{2}$, & -17 & $(9,0,1)$ & 0 \\
& & $0,0,1,0,0,0)$ & $0,0,0,1,0,1)$ & $\left(\frac{5}{13}\right),\left(\frac{6}{13}\right),\left(\frac{9}{13}\right)^{2}$ & & & \\
\hline 17 & 8 & $(1,1,1,1,1,1$, & $(0,0,0,0,0,0$, & $\left(\frac{1}{17}\right)^{2},\left(\frac{4}{17}\right),\left(\frac{5}{17}\right)$, & -23 & $(7,-, 0)$ & 0 \\
& & $0,1,0,1,0,0$, & $0,0,0,0,0,1$, & $\left(\frac{7}{17}\right)^{2},\left(\frac{8}{17}\right),\left(\frac{9}{17}\right)^{2}$ & & & \\
& & $0,0,0,0)$ & $0,0,1,1)$ & & & & \\
\hline 19 & 9 & $(1,1,1,1,1,1$, & $(0,0,0,0,0,0$, & $\left(\frac{1}{19}\right)^{2},\left(\frac{3}{19}\right),\left(\frac{5}{19}\right)^{2}$, & -25 & $(5,-, 0)$ & 0 \\
& & $0,1,1,0,0,1$, & $0,0,0,0,0,0$ & $\left(\frac{7}{19}\right),\left(\frac{9}{19}\right),\left(\frac{13}{19}\right)^{2}$ & & & \\
\hline
\end{tabular}

TABLE 1.

Proof. Let us fix $p$. By Section 4.3 we know the dimension $M_{p}$ of the maximal components of the family of K3 surfaces with a non-symplectic automorphism of order $p$. Since every K3 surface which is the minimal model of a productquotient with group $\mathbb{Z} / p \mathbb{Z}$ admits a non-symplectic automorphism of order $p$ (see Section 3.4), we can bound the number of moduli of the pairs $\left(C_{1}, \mathbf{g}_{1}\right),\left(C_{2}, \mathbf{g}_{2}\right)$ by $M_{p}$, see also Proposition 4.5 .

The first step consists in listing the product-quotient surfaces with $p_{g}=1$ and $q=0$. This is done using the program t1t2PtsSurfaces, giving the group $G \simeq \mathbb{Z} / p \mathbb{Z}$ and the numbers $t 1, t 2$ such that $t 1 \geq 3, t 2 \geq 3$ and $t 1+t 2=m-6$, where $m \leq M_{p}$. Indeed, recall that $t 1$ and $t 2$ are the numbers of branching points of the projections $C_{i} \rightarrow C_{i} / \mathbf{g}_{\mathbf{i}}$ respectively. Then $t 1-3$ and $t 2-3$ are the moduli of the pairs $\left(C_{i}, \mathbf{g}_{\mathbf{i}}\right)$ and $m$ is the dimension of family of the product-quotient surfaces, by Proposition 4.5.

Second step: for every product-quotient surface in the list, one has to check if the minimal model is a K3 surface and has to calculate the fixed locus, determining $(n, g, k+1)$, of the induced automorphism. This is done for every entry in the list exactly as in Example 6.5.

Every member of the family, $\mathcal{F}_{P Q}\left(C_{1}, C_{2}\right)$, of $\mathrm{K} 3$ surfaces which are minimal models of $\left(C_{1} \times C_{2}\right) /\left(\mathbf{g}_{1} \times \mathbf{g}_{2}\right)$, is also a member of a family $\mathcal{M}_{(n, g, k+1)}^{p}$. This implies 
that $\mathcal{F}_{P Q}\left(C_{1}, C_{2}\right) \subset \mathcal{M}_{(n, g, k+1)}^{p}$. For every $(n, g, k+1)$, there exists always a choice of $\left(C_{1}, \mathbf{g}_{\mathbf{1}}\right)$ and $\left(C_{2}, \mathbf{g}_{\mathbf{2}}\right)$ such that $\operatorname{dim}\left(\mathcal{F}_{P Q}\left(C_{1}, C_{2}\right)\right)=\operatorname{dim}\left(\mathcal{M}_{(n, g, k+1)}^{p}\right)$. Since both these families are quasi-projective and irreducible, the generic K3 surface in $\mathcal{M}_{(n, g, k+1)}^{p}$ is the minimal model of a product-quotient surface.

Moreover, we observe that different (up to isomorphism) admissible choices for $\left(C_{1}, \mathbf{g}_{1}\right),\left(C_{2}, \mathbf{g}_{2}\right)$ correspond to the same component $\mathcal{M}_{(n, g, k+1)}^{p}$. In Table 1 we give one example for each component. It is remarkable that one can always construct this example choosing $\left(C_{2}, \mathbf{g}_{2}\right) \simeq\left(D_{p}, \delta_{p}\right)$. In Table 1 one can find: the properties which characterize the pair $\left(C_{1}, \mathbf{g}_{1}\right)$; the singularities of $\left(C_{1} \times D_{p}\right) /\left(\mathbf{g}_{1} \times \delta_{p}\right)$; the value of $K_{X}^{2}$; the fixed locus $(n, g, k+1)$ of the automorphism induced on $S$; and this identifies $\mathcal{M}_{(n, g, k+1)}^{p}$ whose dimension is $m$.

In order to prove the last assertion, we recall that if $S$ is in $\mathcal{M}_{(n, g, k+1)}^{p}$ and has minimal Picard number, then its Picard group is uniquely determined (recall that $p \neq 2)$, we denote it by $L_{(n, g, k+1)}^{p}$. In [2] it is proved that the all K3 surfaces $S$ in $\mathcal{M}_{(n, g, k+1)}^{p}$ and with $N S(S) \simeq L_{(n, g, k+1)}^{p}$ admit certain models. Hence it suffices to prove that all the surfaces admitting such models are product-quotient surfaces. This is done in Section 7.

The quotients $\left(C_{1} \times D_{3}\right) /\left(\mathbf{g}_{1} \times \delta_{3}\right)$ which admit a minimal model which is a K3 surface were already classified in [14], Remark 3.1, and are listed in the first 4 rows of Table 1.

Remark 5.2. The example listed in Table 1 are all the examples obtained as described if $p \leq 7$. For $p \geq 11$ there are other admissible pairs $\left(C_{1}, \mathbf{g}_{1}\right),\left(C_{2}, \mathbf{g}_{2}\right)$, such that $C_{i} \not D_{p}, i=1,2$, which correspond to the components $\mathcal{M}_{(11,0,1)}^{11}$, $\mathcal{M}_{(9,0,1)}^{13}, \mathcal{M}_{(7,-, 0)}^{17}, \mathcal{M}_{(5,-, 0)}^{19}$.

Remark 5.3. A very explicit example of this construction for the K3 surface in line 5 of Table 1 is given in [21].

\section{K3 surfaces which are minimal models of $\left(C_{1} \times C_{2}\right) /(\mathbb{Z} / 2 p \mathbb{Z})$}

In Section 5 we described K3 surfaces which are minimal models of a productquotient surfaces with group $\mathbb{Z} / p \mathbb{Z}$. If $p \leq 11$ we do not get the maximal irreducible components of the moduli space of K3 surfaces with a non-symplectic automorphism of order $p$. In order to find at least one maximal irreducible component of such moduli space, we consider product-quotient surfaces with the group $\mathbb{Z} / 2 p \mathbb{Z}$.

Theorem 6.1. Let $p=3$ (resp. $3<p \leq 13,13<p \leq 19$ ). All the K3 surfaces which are minimal models of $\left(C_{1} \times D_{p}\right) /\left(\mathbf{g}_{1} \times \tau_{p}\right),\left|\mathbf{g}_{1} \times \tau_{p}\right|=2 p$ admit a nonsymplectic automorphism of order $p$ whose fixed locus is one of those listed in Table 2.

Viceversa, let $S$ be a K3 surface generic in the family of the K3 surfaces admitting a non-symplectic automorphism of order $p$ which fixes at least 2 (respectively 1,0$)$ curves. Then $S$ is the minimal model of $\left(C_{1} \times C_{2}\right) /\left(\mathbf{g}_{1} \times \mathbf{g}_{2}\right)$ with 
$\left|\mathbf{g}_{\mathbf{1}} \times \mathbf{g}_{\mathbf{2}}\right|=2 p$ and for each such surface $S$ we can always choose $\left(C_{2}, \mathbf{g}_{\mathbf{2}}\right) \simeq\left(D_{p}, \tau_{p}\right)$ and hence the non-symplectic automorphism on $S$ is always induced by $\mathrm{id} \times \delta_{\mathrm{p}}$. In Table 2 we list an example for each family assuming $\left(C_{2}, \mathbf{g}_{2}\right) \simeq\left(D_{p}, \tau_{p}\right)$.

Moreover, the same holds true for every $K 3$ surface admitting a non-symplectic automorphism of order $p$ which fixes at least 2 (resp. 1,0) curves and with minimal Picard number.

\begin{tabular}{|c|c|c|c|c|c|c|c|}
\hline$p$ & $g\left(C_{1}\right)$ & $\left(\alpha_{1}, \ldots \alpha_{2 p-1}\right)$ & $\left(a_{1}, \ldots, a_{2 p-1}\right)$ & $\operatorname{Sing}\left(C_{1} \times D_{p} / \mathbf{g}_{1} \times \tau_{p}\right)$ & $K_{X}^{2}$ & $(n, g, k+1)$ & $m$ \\
\hline 3 & 25 & $(9,7,5,3,1)$ & $(0,12,0,0,0)$ & $\left(\frac{1}{6}\right)^{12},\left(\frac{1}{3}\right)^{12},\left(\frac{1}{2}\right)^{12}$ & -36 & $(0,5,2)$ & 9 \\
\hline 3 & 22 & $(8,6,4,3,1)$ & $(0,10,2,0,0)$ & $\left(\frac{1}{6}\right)^{10},\left(\frac{1}{3}\right)^{13},\left(\frac{1}{2}\right)^{10}$ & -31 & $(1,4,2)$ & 8 \\
\hline 3 & 19 & $(7,5,3,3,1)$ & $(0,8,4,0,0)$ & $\left(\frac{1}{6}\right)^{8},\left(\frac{1}{3}\right)^{14},\left(\frac{1}{2}\right)^{8}$ & -26 & $(2,3,2)$ & 7 \\
\hline 3 & 16 & $(6,4,2,3,1)$ & $(0,6,6,0,0)$ & $\left(\frac{1}{6}\right)^{6},\left(\frac{1}{3}\right)^{15},\left(\frac{1}{2}\right)^{6}$ & -21 & $(3,2,2)$ & 6 \\
\hline 3 & 17 & $(6,5,3,2,1)$ & $(0,8,0,2,0)$ & $\left(\frac{1}{6}\right)^{8},\left(\frac{1}{3}\right)^{8},\left(\frac{2}{3}\right)^{3},\left(\frac{1}{2}\right)^{8}$ & -24 & $(3,3,3)$ & 6 \\
\hline 3 & 13 & $(5,3,1,3,1)$ & $(0,4,8,0,0)$ & $\left(\frac{1}{6}\right)^{4},\left(\frac{1}{3}\right)^{16},\left(\frac{1}{2}\right)^{4}$ & -16 & $(4,1,2)$ & 5 \\
\hline 3 & 14 & $(5,4,2,2,1)$ & $(0,6,2,2,0)$ & $\left(\frac{1}{6}\right)^{6},\left(\frac{1}{3}\right)^{9},\left(\frac{2}{3}\right)^{3},\left(\frac{1}{2}\right)^{6}$ & -19 & $(4,2,3)$ & 5 \\
\hline 3 & 15 & $(5,4,3,2,1)$ & $(1,7,0,0,0)$ & $\left(\frac{1}{6}\right)^{7},\left(\frac{5}{6}\right),\left(\frac{1}{3}\right)^{7},\left(\frac{2}{3}\right),\left(\frac{1}{2}\right)^{8}$ & -21 & $(4,3,4)$ & 5 \\
\hline 3 & 10 & $(4,2,0,3,1)$ & $(0,2,10,0,0)$ & $\left(\frac{1}{6}\right)^{2},\left(\frac{1}{3}\right)^{17},\left(\frac{1}{2}\right)^{2}$ & -11 & $(5,0,2)$ & 4 \\
\hline 3 & 11 & $(4,3,1,2,1)$ & $(0,4,4,2,0)$ & $\left(\frac{1}{6}\right)^{4},\left(\frac{1}{3}\right)^{10},\left(\frac{2}{3}\right)^{3},\left(\frac{1}{2}\right)^{4}$ & -14 & $(5,1,3)$ & 4 \\
\hline 3 & 12 & $(4,3,2,2,1)$ & $(1,5,2,0,0)$ & $\left(\frac{1}{6}\right)^{5},\left(\frac{5}{6}\right),\left(\frac{1}{3}\right)^{8},\left(\frac{2}{3}\right),\left(\frac{1}{2}\right)^{6}$ & -16 & $(5,2,4)$ & 4 \\
\hline 3 & 7 & $(3,1,0,2,1)$ & $(0,1,8,0,3)$ & $\left(\frac{1}{6}\right),\left(\frac{1}{3}\right)^{13},\left(\frac{1}{2}\right)^{5}$ & -7 & $(6,0,3)$ & 3 \\
\hline 3 & 9 & $(3,2,1,2,1)$ & $(1,3,4,0,0)$ & $\left(\frac{1}{6}\right)^{3},\left(\frac{5}{6}\right),\left(\frac{1}{3}\right)^{9},\left(\frac{2}{3}\right),\left(\frac{1}{2}\right)^{4}$ & -11 & $(6,1,4)$ & 3 \\
\hline 3 & 4 & $(2,0,0,1,1)$ & $(0,0,6,0,6)$ & $\left(\frac{1}{3}\right)^{9},\left(\frac{1}{2}\right)^{8}$ & -3 & $(7,0,4)$ & 2 \\
\hline 3 & 7 & $(2,2,1,1,1)$ & $(1,3,0,2,0)$ & $\left(\frac{1}{6}\right)^{3},\left(\frac{5}{6}\right),\left(\frac{1}{3}\right)^{3},\left(\frac{2}{3}\right)^{4},\left(\frac{1}{2}\right)^{4}$ & -9 & $(7,1,5)$ & 2 \\
\hline 3 & 3 & $(1,0,0,1,1)$ & $(1,0,4,0,3)$ & $\left(\frac{5}{6}\right),\left(\frac{1}{3}\right)^{6},\left(\frac{2}{3}\right),\left(\frac{1}{2}\right)^{5}$ & -2 & $(8,0,5)$ & 1 \\
\hline 3 & 5 & $(1,1,1,1,1)$ & $(2,2,0,0,0)$ & $\left(\frac{1}{6}\right)^{2},\left(\frac{5}{6}\right)^{2},\left(\frac{1}{3}\right)^{2},\left(\frac{2}{3}\right)^{2},\left(\frac{1}{2}\right)^{4}$ & -6 & $(8,1,6)$ & 1 \\
\hline 3 & 1 & $(0,0,0,0,1)$ & $(0,1,2,0,3)$ & $\left(\frac{5}{6}\right),\left(\frac{2}{3}\right)^{4},\left(\frac{1}{2}\right)^{5}$ & 0 & $(9,0,6)$ & 0 \\
\hline 5 & 22 & $\begin{array}{c}(4,2,0,4 \\
2,1,5,3,1)\end{array}$ & $\begin{array}{c}(0,0,6,0 \\
0,0,2,0,0)\end{array}$ & $\begin{array}{c}\left(\frac{1}{10}\right)^{6},\left(\frac{1}{5}\right)^{2} \\
\left(\frac{2}{5}\right)^{6},\left(\frac{3}{5}\right),\left(\frac{1}{2}\right)^{6}\end{array}$ & -28 & $(1,2,1)$ & 4 \\
\hline 5 & 17 & $\begin{array}{c}(3,2,0,3 \\
1,1,4,2,1)\end{array}$ & $\begin{array}{c}(0,0,4,0 \\
2,0,2,0,0)\end{array}$ & $\begin{array}{c}\left(\frac{1}{10}\right)^{4},\left(\frac{1}{5}\right)^{3},\left(\frac{2}{5}\right) \\
\left(\frac{3}{5}\right)^{6},\left(\frac{1}{2}\right)^{4}\end{array}$ & -21 & $(4,1,1)$ & 3 \\
\hline 5 & 13 & $\begin{array}{c}(3,2,1,2 \\
1,1,2,1,0)\end{array}$ & $\begin{array}{c}(0,0,2,2 \\
0,0,2,0,0)\end{array}$ & $\begin{array}{c}\left(\frac{1}{10}\right)^{2},\left(\frac{3}{10}\right)^{2},\left(\frac{1}{5}\right)^{4} \\
\left(\frac{3}{5}\right),\left(\frac{2}{5}\right)^{2},\left(\frac{1}{2}\right)^{4}\end{array}$ & -14 & $(7,1,2)$ & 2 \\
\hline 5 & 12 & $\begin{array}{c}(3,2,1,1 \\
0,2,2,1,0)\end{array}$ & $\begin{array}{c}(0,0,1,1 \\
0,0,6,0,0)\end{array}$ & $\begin{array}{c}\left(\frac{1}{10}\right),\left(\frac{7}{10}\right),\left(\frac{1}{5}\right)^{7} \\
\left(\frac{2}{5}\right)^{3},\left(\frac{3}{5}\right),\left(\frac{4}{5}\right),\left(\frac{1}{2}\right)^{2}\end{array}$ & -13 & $(7,0,1)$ & 2 \\
\hline 5 & 8 & $\begin{array}{c}(2,1,1,1 \\
0,1,1,1,0)\end{array}$ & $\begin{array}{c}(0,0,1,1 \\
0,0,2,2,0)\end{array}$ & $\begin{array}{c}\left(\frac{1}{10}\right),\left(\frac{7}{10}\right),\left(\frac{1}{5}\right)^{3} \\
\left(\frac{2}{5}\right)^{3},\left(\frac{3}{5}\right),\left(\frac{4}{5}\right),\left(\frac{1}{2}\right)^{2}\end{array}$ & -9 & $(10,0,2)$ & 1 \\
\hline 5 & 4 & $\begin{array}{c}(1,1,1,0 \\
0,1,0,0,0)\end{array}$ & $\begin{array}{c}(0,1,0,1 \\
0,0,2,0,0)\end{array}$ & $\begin{array}{c}\left(\frac{3}{10}\right),\left(\frac{9}{10}\right) \\
\left(\frac{1}{5}\right)^{3},\left(\frac{3}{5}\right)^{2},\left(\frac{1}{2}\right)^{2}\end{array}$ & -5 & $(13,0,3)$ & 0 \\
\hline 7 & 19 & $\begin{array}{c}(2,1,3,2,0,3, \\
1,0,3,1,0,2,1)\end{array}$ & $\begin{array}{c}(0,0,0,4,0,0 \\
0,0,2,0,0,0,0)\end{array}$ & $\begin{array}{l}\left(\frac{1}{14}\right)^{4},\left(\frac{4}{7}\right)^{2} \\
\left(\frac{5}{7}\right)^{5},\left(\frac{1}{2}\right)^{4}\end{array}$ & -25 & $(3,1,1)$ & 2 \\
\hline 7 & 13 & $\begin{array}{c}(1,1,2,2,0,1, \\
1,1,2,0,0,1,1)\end{array}$ & $\begin{array}{c}(0,0,0,1,2,1 \\
0,0,0,0,0,0,0)\end{array}$ & $\begin{array}{c}\left(\frac{1}{14}\right)^{2},\left(\frac{11}{14}\right),\left(\frac{5}{14}\right), \\
\left(\frac{1}{7}\right),\left(\frac{5}{7}\right),\left(\frac{3}{7}\right)^{2},\left(\frac{1}{2}\right)^{4}\end{array}$ & -15 & $(8,1,2)$ & 1 \\
\hline 7 & 12 & $\begin{array}{c}(1,1,2,1,0,1, \\
0,1,2,1,0,1,1)\end{array}$ & $\begin{array}{c}(0,0,0,1,1,0 \\
2,0,0,0,2,0,0)\end{array}$ & $\begin{array}{c}\left(\frac{1}{14}\right),\left(\frac{5}{14}\right),\left(\frac{1}{7}\right)^{3} \\
\left(\frac{3}{7}\right),\left(\frac{4}{7}\right),\left(\frac{5}{7}\right)^{3},\left(\frac{1}{2}\right)^{2}\end{array}$ & -14 & $(8,0,1)$ & 1 \\
\hline 7 & 6 & $\begin{array}{c}(0,0,1,0,0,1 \\
0,0,1,1,0,1,1)\end{array}$ & $\begin{array}{c}(1,0,0,1,0,0, \\
2,0,0,0,0,0,0)\end{array}$ & $\begin{array}{l}\left(\frac{1}{14}\right),\left(\frac{9}{14}\right),\left(\frac{1}{7}\right) \\
\left(\frac{3}{7}\right)^{3},\left(\frac{6}{7}\right),\left(\frac{1}{2}\right)^{2}\end{array}$ & -10 & $(13,0,2)$ & 0 \\
\hline 11 & 21 & $\begin{array}{l}(1,0,2,1,2,1,0 \\
2,0,2,1,0,2,0 \\
2,1,0,1,0,2,1)\end{array}$ & $\begin{array}{l}(0,0,0,0,0,3,0, \\
0,1,0,0,0,0,0, \\
0,0,0,0,0,0,0)\end{array}$ & $\begin{array}{c}\left(\frac{1}{22}\right)^{3},\left(\frac{19}{22}\right) \\
\left(\frac{6}{11}\right),\left(\frac{9}{11}\right)^{3},\left(\frac{1}{2}\right)^{4}\end{array}$ & -31 & $(2,1,1)$ & 1 \\
\hline
\end{tabular}




\begin{tabular}{|c|c|c|c|c|c|c|c|}
\hline 11 & 10 & $\begin{array}{l}(1,1,1,1,0,0,0, \\
1,0,0,0,1,1,0, \\
1,1,1,0,0,0,0)\end{array}$ & $\begin{array}{l}(0,0,0,0,0,0,1, \\
0,1,0,0,0,0,0, \\
0,0,0,0,2,0,0)\end{array}$ & $\begin{array}{c}\left(\frac{3}{22}\right),\left(\frac{7}{22}\right),\left(\frac{1}{11}\right)^{2}, \\
\left(\frac{2}{11}\right),\left(\frac{4}{11}\right),\left(\frac{9}{11}\right), \\
\left(\frac{1}{2}\right)^{2}\end{array}$ & -13 & $(11,0,1)$ & 0 \\
\hline 13 & 12 & $\begin{array}{c}(0,0,1,0,1,1,0 \\
1,0,0,0,1,0,0 \\
1,1,1,0,1,0,0 \\
1,0,1,1)\end{array}$ & $\begin{array}{c}(0,0,0,0,0,0,1 \\
0,1,0,0,0,0,0 \\
0,0,0,0,2,0,0 \\
0,0,0,0)\end{array}$ & $\begin{array}{c}\left(\frac{1}{26}\right),\left(\frac{5}{26}\right),\left(\frac{3}{13}\right)^{2} \\
\left(\frac{4}{13}\right),\left(\frac{6}{13}\right),\left(\frac{7}{13}\right) \\
\left(\frac{1}{2}\right)^{2}\end{array}$ & -18 & $(9,0,1)$ & 0 \\
\hline 17 & 16 & $\begin{array}{c}(1,0,1,0,1,0,1 \\
1,0,1,0,0,0,1 \\
1,1,0,0,0,0,1 \\
1,1,0,1,0,0,1 \\
0,1,0,1,0) \\
\end{array}$ & $\begin{array}{c}(0,0,0,0,0,0,0 \\
0,1,1,0,0,0,0 \\
0,0,0,0,0,0,0 \\
0,2,0,0,0,0,0 \\
\quad 0,0,0,0,0)\end{array}$ & $\begin{array}{c}\left(\frac{1}{34}\right),\left(\frac{23}{34}\right), \\
\left(\frac{5}{17}\right)^{2},\left(\frac{7}{17}\right)^{2},\left(\frac{15}{17}\right), \\
\left(\frac{1}{2}\right)^{2}\end{array}$ & -22 & $(7,-, 0)$ & 0 \\
\hline 19 & 18 & $\begin{array}{l}(1,1,1,1,1,0,0, \\
1,1,1,0,0,0,0, \\
0,1,0,0,0,1,1, \\
0,1,1,1,1,1,0,0, \\
0,1,1,0,0,0,0,0)\end{array}$ & $\begin{array}{c}(0,0,0,0,0,0,0,0 \\
0,0,1,0,0,0,1,0 \\
0,0,0,0,0,0,0,0 \\
0,0,0,0,0,0,0 \\
0,0,0,2,0,0)\end{array}$ & $\begin{array}{c}\left(\frac{3}{38}\right),\left(\frac{11}{38}\right),\left(\frac{1}{19}\right)^{2}, \\
\left(\frac{4}{19}\right),\left(\frac{8}{19}\right),\left(\frac{17}{19}\right) \\
\left(\frac{1}{2}\right)^{2}\end{array}$ & -22 & $(5,-, 0)$ & 0 \\
\hline
\end{tabular}

TABLE 2 .

Proof. The proof is analogous to that of Theorem 5.1. We remark that in Table 2 one can find: the properties which characterize the pair $\left(C_{1}, \mathbf{g}_{1}\right)$; the singularities of $\left(C_{1} \times D_{p}\right) /\left(\mathbf{g}_{1} \times \tau_{p}\right)$; the value of $K_{X}^{2}$; the fixed locus $(n, g, k+1)$ of the automorphism induced on $S$; and this identifies $\mathcal{M}_{(n, g, k+1)}^{p}$ whose dimension is $m$.

Corollary 6.2. All the K3 surfaces admitting a non-symplectic automorphism of order $p=3$ (resp. $3<p \leq 13,13<p \leq 19$ ) which fixes at least 2 (resp. 1, 0) curves admit a non-symplectic automorphism of order $2 p$ induced by $\mathrm{id} \times \tau_{\mathrm{p}}$.

The results in Corollary 6.2 were already proved in [8] for $p=3$ and [15] for $p>3$.

Remark 6.3. The example in line 9 of Table 2 was already considered in [10] and is also related to the construction presented in [23].

Remark 6.4. The K3 surfaces given in Table 2 admit an automorphism of order $2 p$, induced by id $\times \tau_{\mathrm{p}}$ (Corollary 6.2) and one of order 2 , induced by id $\times \iota_{\mathrm{p}}$. The fixed locus of these automorphisms can be computed case by case. In Section 7 we compute it in certain cases, by using a projective model of the surfaces.

Example 6.5. As example we completely describe the construction of the $\mathrm{K} 3$ surface in line 19 of Table 2 . We consider the pairs $\left(C_{1}, \mathbf{g}_{\mathbf{1}}\right)$ and $\left(C_{2}, \mathbf{g}_{\mathbf{2}}\right)$ such that $\left|\mathbf{g}_{\mathbf{i}}\right|=10$ and:

- $g\left(C_{1}\right)=22, C_{1} \rightarrow C_{1} /\left\langle\mathbf{g}_{1}\right\rangle$ is branched in 7 points; 6 of them are points of total ramification and the local action of the automorphism near these points is $-\zeta_{5}^{3}$, the seventh point is a ramification point of order 5 and the local action of $\mathbf{g}_{1}{ }^{2}$ is $\zeta_{5}^{3}$; The dimension of the eigenspaces for the induced action in cohomology is $\left(\alpha_{1}, \ldots, \alpha_{9}\right)=(4,2,0,4,2,1,5,3,1)$;

- $C_{2} \simeq D_{5}, \mathbf{g}_{2}=\tau_{5}$ (cf. Example 2.5). 


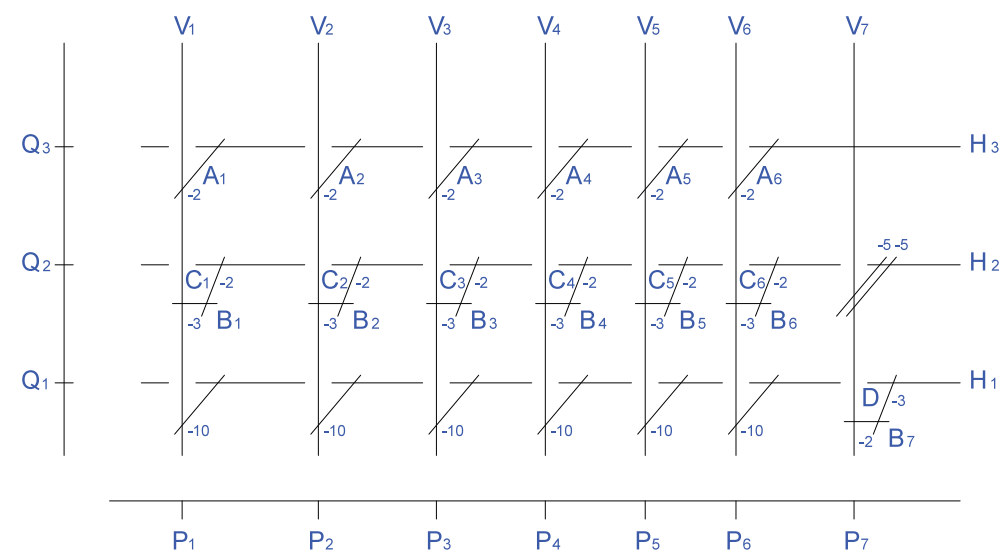

FiguRe 1.

We will denote by $P_{i}, i=1,2,3,4,5,6,7$ the branch points of $C_{1} \rightarrow \mathbb{P}^{1}$, by $Q_{j}$, $j=1,2,3$ the branch points of $C_{2} \rightarrow \mathbb{P}^{1}$ and we assume that $Q_{1}$ is a point of total ramification, $Q_{2}$ is a branch point of order 5 and $Q_{3}$ is a branch point of order 2 .

The singularities of the quotient $\left(C_{1} \times C_{2}\right) /\left(\mathbf{g}_{1} \times \mathbf{g}_{2}\right)$ are:

6 singularities of type $\frac{1}{10}(1,1)$ (over $\left.P_{i} \times Q_{1}, i=1, \ldots, 6\right)$;

6 singularities of type $\frac{1}{5}(1,2)$ (over $\left.P_{i} \times Q_{2}, i=1, \ldots, 6\right)$;

6 singularities of type $\frac{1}{2}(1,1)\left(\right.$ over $\left.P_{i} \times Q_{3}, i=1, \ldots, 6\right)$;

2 singularities of type $\frac{1}{5}(1,1)$ (over $\left.P_{7} \times Q_{2}\right)$;

1 singularity of type $\frac{1}{5}(1,3)$ (over $\left.P_{7} \times Q_{1}\right)$.

The resolution of the singularities is as in Figure 1. From now on we will use the labels as in Figure 1.

By Proposition 3.7 the curves $V_{i}, i=1, \ldots, 7$, are $(-1)$-curves, the curve $H_{1}$ is a $(-1)$-curve, the curve $H_{2}$ is a curve of genus 2 and self intersection -4 and the curve $H_{3}$ is a curve of genus 10 and self intersection -3 .

In order to construct the minimal model $S$, we consider the following contractions:

1) we contract the curves $V_{i}, i=1, \ldots, 7$;

2) we contract the image of the curves $A_{i}, i=1, \ldots, 6$;

3) we contract the image of the curves $B_{i}, i=1, \ldots, 7$;

4) we contract the image of the curves $C_{i}, i=1, \ldots, 6$;

5) we contract the image of the curve $H_{1}$;

$6)$ we contract the image of the curve $D$.

We obtain the surface $S$ as in the Figure 2.

We contracted 28 curves and since the canonical bundle of $X$ has self-intersection $K_{X}^{2}=-28$, we obtain $K_{S}^{2}=0$. Now we verify that the surface $S$ satisfies the hypothesis of Lemma 4.7 and this proves that $S$ is a K3 surface. 


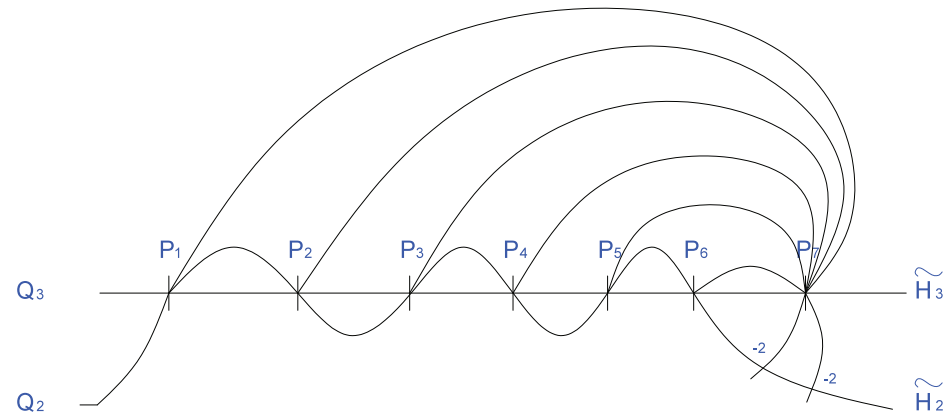

FiguRE 2.

By adjunction, $2 g\left(C_{1}\right)-2=\left(K_{X}-F_{2}\right) F_{2}=K_{X} F_{2}=42$ and $2 g\left(C_{2}\right)-2=$ $\left(K_{X}-F_{1}\right) F_{1}=K_{X} F_{1}=2$. The exceptional divisor $E$ of the blow up $X \rightarrow S$ is

$$
E=\sum_{i=1}^{6}\left(6 V_{i}+3 A_{i}+2 B_{i}+C_{i}\right)+3 V_{7}+2 B_{7}+2 H_{1}+D .
$$

The curves $V_{i}, i=1, \ldots, 6$ are sections of the fibration $\pi_{2}$, the curve $V_{7}$ meets the fiber $F_{1}$ of the same fibration in two points. Since $F_{1} E=F_{1}\left(\sum_{i=1}^{6}\left(6 V_{i}\right)+3 V_{7}\right)=42$, $\left(K_{X}-E\right) F_{1}=K_{X} F_{1}-E F_{1}=0$. Analogously, the curve $H_{1}$ is a section of the fibration $\pi_{1}$. Since $F_{2} E=F_{2} 2 H_{1}=2,\left(K_{X}-E\right) F_{2}=K_{X} F_{2}-E F_{2}=0$.

By Remark 4.9 the fixed locus of $g_{S}$ on $S$ consists of one curve of genus 2, labelled in Figure 2 by $\widetilde{H_{2}}$ and 1 point, labelled in Figure 2 by $P_{7}$.

Since $C_{2}$ is a rigid curve and $C_{1}$ varies in an irreducible 4-dimensional family, we in fact construct an irreducible 4-dimensional family $\mathcal{F}$ of K3 surfaces $S$ with a non-symplectic automorphism of order 5 and fixed locus $(n, g, k+1)=(1,2,1)$. Therefore $\mathcal{F} \subset \mathcal{M}_{(1,2,1)}^{5}$.

We observe that the order 10 automorphism induced on $S$ by id $\times \tau_{5}$ fixes 7 points labelled in Figure 2 by $P_{i}, i=1, \ldots, 7$ and the involution induced on $S$ by $\left(\text { id } \times \tau_{5}\right)^{5}$ fixes one curve of genus 10 , labelled in Figure 2 by $\widetilde{H_{3}}$.

\subsection{Intermediate quotients}

The $2 p: 1$ map $C_{1} \times D_{p} \rightarrow\left(C_{1} \times D_{p}\right) /\left(\mathbf{g}_{1} \times \tau_{\mathbf{p}}\right)$ clearly factorizes through

$$
C_{1} \times D_{p} \stackrel{p: 1}{\longrightarrow}\left(C_{1} \times D_{p}\right) /\left(\mathbf{g}_{\mathbf{1}} \times \tau_{p}\right)^{2} \stackrel{2: 1}{\longrightarrow}\left(C_{1} \times D_{p}\right) /\left(\mathbf{g}_{\mathbf{1}} \times \tau_{p}\right) .
$$

This induces a 2:1 rational map between the minimal model, $Q$, of $\left(C_{1} \times D_{p}\right) /\left(\mathbf{g}_{1} \times\right.$ $\left.\tau_{p}\right)^{2}$ and the K3 surface $S$. In particular $Q$ is a 2-cover of a K3 surface. We observe that $p_{g}(Q) \geq p_{g}(S)$. This immediately implies that the Kodaira dimension $k(Q)$ of $Q$ is non negative. The following examples show that all the other three possibilities, $k(Q)=0,1,2$, are realized by our classification. First we notice that the genus of the quotient $C_{1} / \mathbf{g}_{1}^{2}$ is $\alpha_{p}$ and so $q(Q)=\alpha_{p}$. 
Consider line 18 of Table 2, corresponding to the quotient $\left(D_{3} \times D_{3}\right) /\left(\tau_{3}^{5} \times \tau_{3}\right)$. The quotient $\left(D_{3} \times D_{3}\right) /\left(\tau_{3}^{5} \times \tau_{3}\right)^{2}$ is isomorphic to $\left(D_{3} \times D_{3}\right) /\left(\delta_{3} \times \delta_{3}^{2}\right)$. The minimal model of such a surface is described in [34] (see Examples 3.8 and 2.5, and the fourth line of Table 1) and is a K3 surface. In particular in this case $k(Q)=0$.

Let us consider the line 9 of Table 2. The map $C_{1} \rightarrow C_{1} / \mathbf{g}_{1}^{2} \simeq \mathbb{P}^{1}$ is branched in 12 points and an equation of $C_{1}$ is $w^{3}=p_{12}(t)$ where $p_{12}(t)$ is a polynomial with 12 simple roots. With the same method we will apply in Section 7, case $p=3$, we obtain the equation $y^{2}=x^{3}+p_{12}^{2}(t)$ of $\left(C_{1} \times D_{p}\right) /\left(\mathbf{g}_{1} \times \tau_{p}\right)^{2}$. So the surface $Q$ admits an elliptic fibration, its birational invariant are $q(Q)=0$, $p_{g}(Q)=3=\alpha_{2}+\alpha_{5}$, and we obtain $k(Q)=1$.

Let us consider the line 19 of Table 2 . It corresponds to the quotient $\left(C_{1} \times D_{5}\right) /$ $\left(\mathbf{g}_{1} \times \tau_{5}\right)$ where $g\left(C_{1}\right)=22$. Let $Y$ be the minimal resolution of $\left(C_{1} \times D_{5}\right) /\left(\mathbf{g}_{1} \times \tau_{5}\right)^{2}$. As in Example 6.5, one proves that the singularities of $\left(C_{1} \times D_{5}\right) /\left(\mathbf{g}_{1} \times \tau_{5}\right)^{2}$ are 10 singularities of type $\frac{1}{5}(1,1), 12$ singularities of type $\frac{1}{5}(1,2)$ and 2 singularities of type $\frac{1}{5}(1,3)$. The computation of $K_{Y}^{2}$ can be done as explained in Remark $3.3(4)$ and it gives $K_{Y}^{2}=10>0$. Since $Q$ is the minimal model of $Y, K_{Q}^{2} \geq K_{Y}^{2}$ and we conclude that $K_{Q}^{2}>0, Q$ is a surface of general type, and so $k(Q)=2$.

\section{Equations}

\subsection{Automorphisms of order $p=3$}

In Proposition 4.2 of [1] it is proved that every K3 surface admitting a non-symplectic automorphism of order 3 fixing at least two curves is in fact an isotrivial elliptic fibration with generic fiber isomorphic to the elliptic curve $E_{\zeta_{3}}$ with complex multiplication of order 3. Indeed, every such a K3 surface is described as an elliptic K3 surface with an equation of type $y^{2}=x^{3}+f_{12}(t)$. In view of our construction this is very natural: we proved that every such a K3 surface is the minimal model of the quotient $\left(C_{1} \times D_{3}\right) /\left(\mathbf{g}_{1} \times \tau_{3}\right)$ where $D_{3} \simeq E_{\zeta_{3}}$ and $\tau_{3}$ are described in Example 2.5 and $\left(C_{1}, \mathbf{g}_{1}\right)$ varies. The maximal component is obtained by $\left(C_{1}, \mathbf{g}_{1}\right)$ as in the first line of Table 2 . In this case $C_{1}$ is a $6: 1$ cover of $\mathbb{P}^{1}$ whose ramification consists of 12 points of order 6 . An equation of $C_{1}$ is $w^{6}=$ $f_{12}(t)$, where $\operatorname{deg}\left(f_{12}(t)\right)=12$ and $f_{12}(t)$ does not have multiple roots. The local action near the fixed points is $-\zeta_{3}^{2}$ (see Table 2) and thus we can assume that the automorphism $\mathbf{g}_{\mathbf{1}}$ is $\mathbf{g}_{\mathbf{1}}:(w, t) \mapsto\left(-\zeta_{3}^{2} w, t\right)$. The new functions $x:=u w^{2}$, $y:=v w^{3}$ and $t$ are invariant for $\mathbf{g}_{1} \times \tau_{3}$ and satisfy the equation

$$
y^{2}=x^{3}+f_{12}(t) .
$$

Moreover, if $W$ is the surface defined by this equation, then the generic fiber of the map $C_{1} \times D_{3} \rightarrow W$ consists of 6 points, thus we have the following commutative diagram:

$$
\begin{array}{ccc}
6: 1 \swarrow & C_{1} \times D_{3} & \\
W & & \searrow 6: 1 \\
W & -\rightarrow & \left(C_{1} \times D_{3}\right) /\left(\mathbf{g}_{1} \times \tau_{3}\right)
\end{array}
$$


which shows that $W$ and $S$ are birational and so $W$ is a singular model of the K3 surface $S$. This construction was also considered in Example 3.11 of [16].

More in general, the curve $C_{1}$ has an equation of type $w^{6}=f_{12}(t)$, where $f_{12}$ does not admit roots with multiplicity greater than 5 and there exists no a polynomial $h(t)$ such that either $f_{12}(t)=h^{2}(t)$ or $f_{12}(t)=h^{3}(t)$. If some of the roots of $f_{12}(t)$ have multiplicity higher than 1 , then the fixed locus of id $\times \delta_{3}$ changes and we obtain a member of a more special family (cf. lines from 2 to 18 of Table 2).

We saw in Section 5 that certain K3 surfaces admitting a non-symplectic automorphism of order 3 , can be obtained as quotient of $\left(C_{1} \times D_{3}\right)$ by an automorphism of order $3, \mathbf{g}_{1} \times \delta_{3}$. So we obtain a different equation for these K3 surfaces. Indeed, in this case one can assume $C_{1}$ to have the following equation $w^{3}=f_{6}(t)$, such that $f_{6}(t)$ does not admit roots with multiplicity greater than 2 . In a very similar way as before this gives the following equation for the quotient surface:

$$
y^{2}=x^{3}+f_{6}(t)^{2} .
$$

These equations were already considered in [14] and [15].

In [2] it is proved that every $\mathrm{K} 3$ surface in $\mathcal{M}_{(n, g, k+1)}^{3}$ and with minimal Picard number has the following equation: $y^{2}=x^{3}+f_{12}(t)$. This is exactly the one of the product-quotient $\left(C_{1} \times D_{3}\right) /\left(g_{1} \times \tau_{3}\right)$ with $C_{1}$ given by $w^{6}=f_{12}(t)$. Observe that also the equation of $\left(C_{1} \times D_{3}\right) /\left(g_{1} \times \delta_{3}\right)$ is of this type. This concludes the proofs of Theorems 5.1 and 6.1 in case $p=3$.

\subsection{Automorphisms of order $p=5$}

In Theorem 6.1 and Table 2 we proved that the K3 surfaces admitting a nonsymplectic automorphism of order 5 with at least one curve in the fixed locus are the minimal models of quotients $\left(C_{1} \times D_{5}\right) /\left(\mathbf{g}_{1} \times \tau_{5}\right)$ for a certain choices of the pair $\left(C_{1}, \mathbf{g}_{\mathbf{1}}\right)$. In particular the maximal component (with fixed locus $(n, g(C), k+1)=$ $(1,2,1))$ is obtained choosing $C_{1}$ to be a $10: 1$ cover of $\mathbb{P}^{1}$ branched along 6 points of order 10 and 1 point of order 5 . An equation of $C_{1}$ is $w^{10}=f_{6}(t)$ where $\operatorname{deg}\left(f_{6}(t)\right)=6$ and $f_{6}(t)$ does not have multiple roots (we are assuming the branch point of order 5 is at infinity). The local action near the fixed points is $-\zeta_{5}^{3}$ (see Table 2) and so we can assume that the automorphism $\mathbf{g}_{\mathbf{1}}$ is $\mathbf{g}_{\mathbf{1}}:(w, t) \mapsto$ $\left(-\zeta_{5}^{3} w, t\right)$. The functions $x:=u w^{2}, y:=v w^{5}$ and $t$ are invariant under $\mathbf{g}_{1} \times \tau_{5}$ and satisfy the equation

$$
y^{2}=x^{5}+f_{6}(t) .
$$

As in the case $p=3$, one shows that this gives in fact a (singular) model of the K3 surface $S$. The equation exhibits $S$ as double cover of $\mathbb{P}_{[x, t]}^{2}$ branched along the (non homogenous) sextic $x^{5}+f_{6}(t)=0$. A similar model for this K3 surface is described in Example 5.1 of [2], where the relation with the curves $C_{1}$ and $D_{5}$ was not observed.

More in general, we observe that every curve $C_{1}$ in Table 2 admits an equation of the type $w^{10}=f_{6}(t)$ with $f_{6}(t)$ which is not a square, such that $\mathbf{g}_{1}:(w, t) \rightarrow$ $\left(-\zeta_{5}^{2} w, t\right)$. If $f_{6}(t)$ is generic we find the previous equation and so the maximal 
component of the moduli space of K3 surfaces admitting a non-symplectic automorphism of order 5 fixing at least one curve. Specializations of the polynomial $f_{6}(t)$ induce specializations of the K3 surface $S$. For example the line 20 of Table 2 corresponds to the curve $C_{1}$ given by $w^{10}=t^{2} g_{4}(t), \operatorname{deg}\left(g_{4}(t)\right)=4$, and $g_{4}$ does not have multiple roots. The corresponding K3 surface is the double cover of $\mathbb{P}_{[x, t]}^{2}$ branched along the sextic $x^{5}+t^{2} g_{4}(t)=0$, which has a singular point of type $A_{4}$ in the point $(x, t)=(0,0)$.

Every K3 surface that is the double cover of $\mathbb{P}^{2}$ branched along a sextic can be viewed as a hypersurface in the weighted projective space $W \mathbb{P}(3,1,1,1)$. In particular, the homogeneous equation of $S$ can be written as $y^{2}=x^{5} s+f_{6}(t: s)$, where $(y: x: s: t)$ are the homogeneous coordinates of $W \mathbb{P}(3,1,1,1)$ ( $y$ is the coordinate of weight 3 ). This remark will be useful in view of the equations we found in cases $p=7,11$.

In [2] it is proved that every $\mathrm{K} 3$ surface in $\mathcal{M}_{(n, g, k+1)}^{5}$ and minimal Picard number is a double cover of $\mathbb{P}_{(x: s: t)}^{2}$ with following equation $y^{2}=x^{5} s+f_{6}(t: s)$. This is exactly the one of the product-quotient $\left(C_{1} \times D_{5}\right) /\left(g_{1} \times \tau_{5}\right)$ with $C_{1}$ given by $w^{5}=f_{6}(t)$. Observe that also the equation of $\left(C_{1} \times D_{5}\right) /\left(g_{1} \times \delta_{5}\right)$ is of this type. This concludes the proofs of Theorems $5.1,6.1$ in case $p=5$.

The automorphism $\tau_{5}$ (resp. $\delta_{5}$ ) on $D_{5}$ induces the non-symplectic automorphism id $\times \tau_{5}\left(\right.$ resp. id $\left.\times \delta_{5}\right)$ of order 10 (resp. 5) on the K3 surface $S$ which acts on the coordinates $(x, y, t)$ as $(x, y, t) \rightarrow\left(\zeta_{5}^{4} x,-y, t\right)\left(\operatorname{resp} .(x, y, t) \rightarrow\left(\zeta_{5}^{4} x, y, t\right)\right)$. The fixed locus of id $\times \delta_{5}$ consists of one curve of genus 2 if $f_{6}(t)$ is generic, and specializes to different fixed locus when $f_{6}(t)$ specializes (see also [2], Example 5.1).

We observe that the non-symplectic automorphism $\left(i d \times \tau_{5}\right)^{5}$ of order 2 is exactly the cover involution of the double cover of $\mathbb{P}^{2}$ and this allows one to compute easily its fixed locus.

We saw in Section 5 that certain K3 surfaces admitting a non-symplectic automorphism of order 5 , can be obtained from the quotient $\left(C_{1} \times D_{5}\right) /\left(\mathbf{g}_{1} \times \delta_{5}\right)$. So we obtain a different equation for these K3 surfaces. The surfaces obtained in this way are listed in Table 1 . In the case of the 1-dimensional and 0-dimensional families the equation of the curve $C_{1}$ is $w^{5}=f_{3}(t), \operatorname{deg}\left(f_{3}(t)\right)=3$ and $f_{3}$ is not a cube, and the automorphism is $\mathbf{g}_{1}:(w, t) \rightarrow\left(\zeta_{5}^{3} w, t\right)$. The functions $x:=u w^{2}$, $y:=v w^{5}$ and $t$ are invariant and give a (singular) model of the K3 surface $S$, with equation $y^{2}=x^{5} s+f_{3}^{2}(t)$.

\subsection{Automorphisms of order $p=7$}

In Theorem 6.1 and Table 2, we proved that the K3 surfaces admitting a nonsymplectic automorphism of order 7 with at least one curve in the fixed locus are the minimal model of the quotient $\left(C_{1} \times D_{7}\right) /\left(\mathbf{g}_{1} \times \tau_{7}\right)$ for a certain choice of the pair $\left(C_{1}, \mathbf{g}_{1}\right)$. In particular, the maximal component (with fixed locus $(n, g(C), k+$ $1)=(3,1,1))$ is obtained choosing $C_{1}$ to be a $14: 1$ cover of $\mathbb{P}^{1}$ branched along 4 points of order 14 and 1 point of order 7 . An equation of $C_{1}$ is $w^{14}=t(t-1)(t-$ $\left.\lambda_{1}\right)\left(t-\lambda_{2}\right)$. The local action near the fixed points is $-\zeta_{7}^{4}$ (see Table 2 ) and thus the automorphism is $\mathbf{g}_{1}:(w, t) \mapsto\left(-\zeta_{7}^{4} w, t\right)$. The functions $x:=u w^{2}, y:=v w^{7}, t$ 
are invariant for $\mathbf{g}_{\mathbf{1}} \times \tau_{7}$ and satisfy the equation $y^{2}=x^{7}+t(t-1)\left(t-\lambda_{1}\right)\left(t-\lambda_{2}\right)$. As in case $p=3$, one shows that this gives in fact a (singular) model of the K3 surface $S$ : the equation can be homogeneized to

$$
y^{2}=x^{7} s+t\left(t-s^{2}\right)\left(t-\lambda_{1} s^{2}\right)\left(t-\lambda_{2} s^{2}\right) \subset W \mathbb{P}(4,2,1,1)_{(y: t: x: s)} .
$$

In order to show that equation (7.1) corresponds in fact to a (singular model of a) K3 surface we observe that the surface defined by (7.1) is well formed (Definition 6.9 in [12]) and quasismooth (Definition 6.3 in [12]). If a hypersurface $Z$ of degree $d$ in a weighted projective space $W \mathbb{P}\left(a_{0}, a_{1}, a_{2}, a_{3}\right)$ is well formed and quasismooth, then the adjunction formula generalizes and the canonical sheaf is $\omega_{Z} \simeq \mathcal{O}_{Z}\left(d-\sum_{i=0}^{3} a_{i}\right)$ (cf. Paragraph 6.14 in [12]). In particular, if $d=\sum_{i=0}^{3} a_{i}$, then $Z$ is a K3 surface and so the surface defined by (7.1) is a singular model of a K3 surface.

We recall that the generic hypersurface of degree 8 in $W \mathbb{P}(4,2,1,1)$ is a singular model of a K3 surface ([31], Section 4.5) with two singularities of type $\frac{1}{2}(1,1)$ at the points $(1: 1: 0: 0),(-1: 1: 0: 0)$. The surface defined by $(7.1)$ (which is not general) has no other singular points.

Remark 7.1. Observe that the K3 surface described by the equation $y^{2}=x^{7} s+$ $t\left(t-s^{2}\right)\left(t-\lambda_{1} s^{2}\right)\left(t-\lambda_{2} s^{2}\right)$ admits an elliptic fibration, induced by the pencil of rational curves $x=\mu s$ in $W \mathbb{P}(2,1,1)_{(t: s: x)}$, whose fiber over $\bar{\mu}$ is isomorphic to $y^{2}=\overline{\mu^{7}} s^{4}+t(t-s)\left(t-\lambda_{1} s\right)\left(t-\lambda_{2} s\right)$. By elementary transformations one can bring it to the following Weierstrass form $Y^{2}=X^{3}+\left(\alpha+\beta \mu^{7}\right) X+\left(\mu^{7}-1\right)$. Viceversa every K3 surfaces admitting an elliptic fibration described by the equation $Y^{2}=$ $X^{3}+\left(\alpha+\beta \mu^{7}\right) X+\left(\mu^{7}-1\right)$ can be described as a hypersurface in $W \mathbb{P}(4,2,1,1)$ with equation (7.1) by the inverse transformation.

In [2] it is proved that every $\mathrm{K} 3$ surface in $\mathcal{M}_{(n, g, k+1)}^{3}$ and with minimal Picard number admits an elliptic fibration with the following Weierstrass equation: $y^{2}=x^{3}+\left(a+b t^{7}\right) x+\left(t^{7}-1\right)$. We proved that the product-quotient surfaces $\left(C_{1} \times D_{7}\right) /\left(g_{1} \times \tau_{7}\right)$ has the equation (7.1). By Remark 7.1, every surface with Weierstrass equation $y^{2}=x^{3}+\left(a+b t^{7}\right) x+\left(t^{7}-1\right)$ admits also a model as the one given by (7.1) and so is a product-quotient surface $\left(C_{1} \times D_{7}\right) /\left(g_{1} \times \tau_{7}\right)$, with $C_{1}$ given by $w^{14}=f_{4}(t)$. Observe that also the equation of $\left(C_{1} \times D_{7}\right) /\left(g_{1} \times \delta_{7}\right)$ is of this type. This concludes the proofs of Theorems 5.1 and 6.1 in case $p=7$.

The automorphism induced on $S$ by id $\times \tau_{7}$ acts on the coordinates of $W \mathbb{P}(4,2$, $1,1)$ in the following way: $(y: t: x: s) \mapsto\left(-y: t: \zeta_{7}^{6} x: s\right)$. It has order 14 and its fixed locus consists of 5 points: $(0: 0: 1: 0),(0: 0: 0: 1),(0: 1: 0: 1)$, $\left(0: \lambda_{1}: 0: 1\right),\left(0: \lambda_{2}: 0: 1\right)$. The singular points of $W \mathbb{P}(4,2,1,1)$ are switched by the automorphism. The fixed locus of the non-symplectic automorphism of order 7 induced by id $\times \delta_{7}$ consists of the point $(0: 0: 1: 0)$ and of the curve $y^{2}=t(t-$ $\left.s^{2}\right)\left(t-\lambda_{1} s^{2}\right)\left(t-\lambda_{2} s^{2}\right) \subset W \mathbb{P}(4,2,1)$. The well formed expression (cf. Definition 5.11 in [12]) of this curve is $y^{2}=t(t-s)\left(t-\lambda_{1} s\right)\left(t-\lambda_{2} s\right) \subset W \mathbb{P}(2,1,1)$ (see Lemma 5.7 in [12]) and the genus of such a curve is 1 (e.g., Corollary 3.4.4 in [9]).

We observe that the singular points of $W \mathbb{P}(4,2,1,1)$ are contained in the fixed locus of the automorphism. From (7.1) one sees that $S$ is a $2: 1$ ramified cover of $W \mathbb{P}(2,1,1)$, with branch locus given by $x^{7} s+t\left(t-s^{2}\right)\left(t-\lambda_{1} s^{2}\right)\left(t-\lambda_{2} s^{2}\right)=0$. The 
weighted projective plane $W \mathbb{P}(2,1,1)$ has a natural embedding in $\mathbb{P}^{3}$ with coordinates $\left(x_{0}: x_{1}: x_{2}: x_{3}\right)=\left(t: x^{2}: s^{2}: x s\right)$, whose image is a cone $Q$ of equation $x_{3}^{2}=x_{1} x_{2}$. The branch locus of the covering is now given by the intersection of $Q$ and the curve $x_{1}^{3} x_{3}+x_{0}\left(x_{0}-x_{2}\right)\left(x_{0}-\lambda_{1} x_{2}\right)\left(x_{0}-\lambda_{2} x_{2}\right)=0$, which does not pass through the vertex of $Q$. The automorphism descends to $\mathbb{P}^{3}$ with the action $\left(x_{0}: x_{1}: x_{2}: x_{3}\right) \mapsto\left(x_{0}: \zeta_{7}^{2} x_{1}: x_{2}: \zeta_{7} x_{3}\right)$. The fixed locus is the isolated point $(0: 1: 0: 0)$ and the curve $x_{1}=x_{3}=0$, which passes through the vertex of $Q$. We blow up the vertex of $Q$ introducing a copy of $\mathbb{P}^{1}$. The induced automorphism leaves invariant the exceptional divisor $E$ and fixes the strict transform of the fixed curve $B$. Since it restricts to an automorphism of $E$, it fixes two points on it, one of them is $E \cap B$. Above the other fixed point on $E$ we find two fixed point on $S$.

The fixed locus of the non-symplectic involution id $\times \iota$ is the curve $x^{7} s+t(t-$ $\left.s^{2}\right)\left(t-\lambda_{1} s^{2}\right)\left(t-\lambda_{2} s^{2}\right) \subset W \mathbb{P}(2,1,1)$. This is a curve of genus 9 in $W \mathbb{P}(2,1,1)$ by Corollary 3.4.4 in [9].

In Table 1 we showed that certain K3 surfaces admitting a non-symplectic automorphism of order 7 , can be obtained from the quotient $\left(C_{1} \times D_{7}\right) /\left(\mathbf{g}_{1} \times \delta_{7}\right)$. In the case of the 0-dimensional family the equation of the curve $C_{1}$ is $w^{7}=t(t-1)$ and the automorphism is $\mathbf{g}_{\mathbf{1}}:(w, t) \rightarrow\left(\zeta_{7}^{4} w, t\right)$. The functions $x:=u w^{2}, y:=v w^{7}$ and $t$ are invariant and gives a (singular) model of the K3 surface $S$, with equation $y^{2}=x^{7} s+t^{2}\left(t-s^{2}\right)^{2} \subset W \mathbb{P}(4,2,1,1)_{(y: t: x: s)}$.

\subsection{Automorphisms of order $p=11$}

If $p=11$, one can obtain an equation for a (singular model) of $S$, minimal model of $\left(C_{1} \times D_{11}\right) /\left(\mathbf{g}_{1} \times \tau_{11}\right)$, as in cases $p=3,5,7$ : an equation for the curve $C_{1}$ is $w^{11}=t(t-1)(t-\lambda)$ (where if $\lambda \neq 0,1$ the curve $C_{1}$ is the one described in line 27 of Table 2 , if either $\lambda=1$ or $\lambda=0$, the curve $C_{1}$ is the one described in line 28 of Table 2) and the automorphism $\mathbf{g}_{1}:(w, t) \rightarrow\left(-\zeta_{11}^{6}, t\right)$. An equation of $S$ is $y^{2}=x^{11} s-t\left(t-s^{4}\right)\left(t-\lambda s^{4}\right)$ where $y:=v w^{11}, t, x:=u w^{2}$ and $s$ are coordinates of the weighted projective space $W \mathbb{P}(6,4,1,1)$. As in case $p=7$, one shows that this equation define in fact a singular model $W$ of a K3 surface. The surface $W$ is singular in the point $(1: 1: 0: 0)$.

As in the previous cases, in order to conclude the proofs of Theorems 5.1 and 6.1 it suffices to show that every K3 surface with equation the elliptic fibration $y^{2}=x^{3}+a x+t^{11}$ (cf. Example 7.1 in [2]) admits in fact the equation $y^{2}=$ $x^{11} s-t\left(t-s^{4}\right)\left(t-\lambda s^{4}\right) \subset W \mathbb{P}(6,4,1,1)$. This can be done as in Remark 7.1

The automorphism id $\times \tau_{11}$ induces the non-symplectic automorphism $(y: t$ : $x: s) \mapsto\left(-y: t: \zeta_{11}^{10} x: s\right)$ on the surface $W$ whose fixed locus consists of the points $(0: 0: 0: 1),(0: 1: 0: 1),(0: \lambda: 0: 1)$, and $(0: 0: 1: 0)$ (which are all distinct if $\lambda \neq 0$ and $\lambda \neq 1)$. The point $(0: 0: 1: 0)$ is a singular point of type of the surface.

The automorphism id $\times \delta_{11}$ induces the non-symplectic automorphism $(y: t$ : $x: s) \mapsto\left(y: t: \zeta_{11}^{10} x: s\right)$ on the surface $W$ whose fixed locus consists of the point $(0: 0: 1: 0)$ and of the curve $y^{2}=t\left(t-s^{4}\right)\left(t-\lambda s^{4}\right) \subset W \mathbb{P}(6,4,1)$. The well formed expression of this curve is $y^{2}=t\left(t-s^{2}\right)\left(t-\lambda s^{2}\right) \subset W \mathbb{P}(3,2,1)$ which is quasismooth if $\lambda \neq 0, \lambda \neq 1$. In this case the genus of the curve is 1 (see Theorem 7.2 in [12]). 
The automorphism id $\times \iota$ induces the non-symplectic involution $(y: t: x: s)$ $\mapsto(-y: t: x: s)$ on the surface $W$ whose fixed locus consists of the curve $x^{11} s-t\left(t-s^{4}\right)\left(t-\lambda s^{4}\right) \subset W \mathbb{P}(4,1,1)$ whose genus is 10 , if $\lambda \neq 0, \lambda \neq 1$ (see Theorem 7.2 in [12]).

\section{Moduli of K3 surfaces}

By Theorems 5.1 and 6.1, certain components $\mathcal{M}_{(n, g(C), k+1)}^{p}$ of the moduli space of the K3 surfaces with an automorphism of order $p$ coincide with certain moduli spaces $\mathcal{F}_{P Q}\left(C_{1}, D_{p}\right)$ of the K3 surfaces which are minimal models either of the quotients $\left(C_{1} \times D_{p}\right) /\left(\mathbf{g}_{1} \times \delta_{p}\right)$ or of the quotients $\left(C_{1} \times D_{p}\right) /\left(\mathbf{g}_{1} \times \tau_{p}\right)$. Since both $D_{p} \rightarrow D_{p} / \delta_{p} \simeq \mathbb{P}^{1}$ and $D_{p} \rightarrow D_{p} / \tau_{p} \simeq \mathbb{P}^{1}$ are branched in 3 points, the parameters of the family depend only on the parameters of the curve $C_{1}$. In particular the dimension of the family of K3 surfaces is $r-3$, where $r=\sum a_{i}$ is the number of ramification points of the cover $C_{1} \rightarrow C_{1} / \mathrm{g}_{1} \simeq \mathbb{P}^{1}$, cf. Proposition 4.5. Here we describe the relation between the moduli of the curve $C_{1}$ and the moduli of the surface $S$. In particular we relate the variation of the Hodge structure of weight 2 of $S$ with the one of $H^{1}\left(C_{1}, \mathbb{Q}\right)$.

A particular case is the one with $p=3$ and the quotient $\left(C_{1} \times D_{3}\right) /\left(\mathbf{g}_{1} \times \delta_{3}\right)$. In this case the variation of the Hodge structure of $S$ (and of a Calabi-Yau 3-fold constructed from $S$ and $D_{3}$ ) is described in [14]. Moreover, if the family is 1dimensional, the Picard-Fuchs equation of the surface $S$ is found from the one of the curve $C_{1}$ (cf. Section 2.5 in [14]).

We now assume $S$ to be the minimal model of $\left(C_{1} \times D_{p}\right) /\left(\mathbf{g}_{1} \times \delta_{p}\right)$. By construction $S$ admits a non-symplectic automorphism $\mathbf{g}_{S}$ induced by id $\times \delta_{\mathrm{p}}$. The moduli space of the K3 surfaces $S$ obtained in such a way is determined by the variation of the period of $S$ in a certain eigenspace $H^{2}(S, \mathbb{C})_{\zeta_{p}^{j}}$, cf. Section 4.3. Indeed, the choice of the period of $S$ determines the Hodge structure of $H^{2}(S, \mathbb{C})$ completely.

Proposition 8.1. Let $S$ be a generic $K 3$ surface in the family $\mathcal{F}_{P Q}\left(C_{1} \times D_{p}\right)$ of the surfaces minimal models of $\left(C_{1} \times D_{p}\right) /\left(\mathbf{g}_{\mathbf{1}} \times \delta_{p}\right)$ listed in Table 1. The weight 2 Hodge structure of the transcendental lattice of $S, T_{S} \otimes \mathbb{Q}$, is induced by the weight 1 Hodge structure of $H^{1}\left(C_{1}, \mathbb{Q}\right)$. In particular, the half twist $\left(T_{S} \otimes \mathbb{Q}\right)_{1 / 2}$ is $H^{1}\left(C_{1}, \mathbb{C}\right)$. As a consequence the dimension of the family $\mathcal{F}_{P Q}\left(C_{1} \times D_{p}\right)$ is $2 g\left(C_{1}\right) /(p-1)-1$.

Proof. Since $S$ is generic, the transcendental lattice of $S$ carries a weight 2 Hodge structure of type $(1,(p-1)(m+1)-2,1)$. Since the K3 surface $S$ admits a nonsymplectic automorphism $\mathbf{g}_{S}$, the Hodge structure of $T_{S} \otimes \mathbb{Q}$ is of CM-type with the field $K \simeq \mathbb{Q}\left(\zeta_{p}\right)$ (cf. [17]). In order to perform a half twist on the Hodge structure one has to fix a CM-type, i.e. a set $\Sigma$ of $(p-1) / 2$ distinct embeddings of $K$ in $\mathbb{C}$ with the property that no two of them are conjugate. By abuse of notation, we put $\Sigma=\left\{\zeta_{p}, \ldots, \zeta_{p}^{(p-1) / 2}\right\}$ and so $\bar{\Sigma}=\left\{\zeta_{p}^{(p+1) / 2}, \ldots, \zeta_{p}^{p-1}\right\}$. 
The eigenspaces decomposition (for the action of $\delta_{p}$ ) of $H^{1}\left(D_{p}\right)$ consists of $p-1$ vector spaces of dimension 1 . Therefore $K_{-1 / 2} \simeq H^{1}\left(D_{p}\right)$ as Hodge structure of weight 1 , where $K_{-1 / 2}$ is the negative half twist of $K$ (see Section 1.4 in [17])

Let us denote by $\nu: C_{1} \times D_{p} \rightarrow S$ the map induced by the quotient map. The pull-back $\nu^{*}$ maps $T_{S} \otimes \mathbb{Q}$ in the $\left(\mathbf{g}_{1} \times \delta_{p}\right)$-invariants in $H^{1}(C, \mathbb{Q}) \otimes H^{1}\left(D_{p}, \mathbb{Q}\right)$. For dimensional reason,

$$
T_{S} \otimes \mathbb{Q} \simeq \nu^{*}\left(T_{S} \otimes \mathbb{Q}\right)=\left(H^{1}(C, \mathbb{Q}) \otimes_{\mathbb{Q}} H^{1}\left(D_{p}, \mathbb{Q}\right)\right)^{\mathbf{g}_{1} \times \delta_{p}} .
$$

Let us consider the half twist of both the members of the above equation:

$$
\left(T_{S} \otimes \mathbb{Q}\right)_{1 / 2} \simeq\left(\left(H^{1}(C, \mathbb{Q}) \otimes_{\mathbb{Q}} H^{1}\left(D_{p}, \mathbb{Q}\right)\right)^{\mathbf{g}_{1} \times \delta_{p}}\right)_{1 / 2}
$$

In order to compute the second member of (8.1), we first consider the $\left(\mathbf{g}_{1} \times \delta_{p}\right)$ invariant subspace of $H^{1}\left(C_{1}, \mathbb{C}\right) \otimes H^{1}\left(D_{p}, \mathbb{C}\right) \subset H^{2}\left(C_{1} \times D_{p}, \mathbb{C}\right)$. We recall that $H^{1,0}\left(D_{p}\right)_{\zeta_{p}^{i}}$ is an eigenspace of dimension 1 if $i \leq(p-1) / 2$, and is trivial if $i>(p-1) / 2$. By the fact that $p_{g}(S)=1$, the pair $\left(C_{1}, \mathbf{g}_{1}\right)$ is such that there exists only one value $\bar{h}$ such that $\bar{h}>(p-1) / 2$ and $H^{1,0}\left(C_{1}\right)_{\zeta_{p}^{\bar{h}}}$ is non zero. So the Hodge decomposition of an eigenspace $H^{1}\left(C_{1}\right)_{\zeta_{p}^{k}}$ is trivial, in the sense that $H^{1}\left(C_{1}\right)_{\zeta_{p}^{k}}=H^{a, b}\left(C_{1}\right)_{\zeta_{p}^{k}}$ with $(a, b) \in\{(1,0),(0,1)\}$, except for $k=\bar{h}$. Hence we obtain:

$$
\begin{aligned}
\left(\left(H^{1}(C, \mathbb{C}) \otimes H^{1}\left(D_{p}, \mathbb{C}\right)\right)^{\mathbf{g}_{1} \times \delta_{p}}\right)=\sum_{i=1}^{p-1}\left(\left(H^{1}\left(C_{1}, \mathbb{C}\right)_{\zeta_{p}^{i}} \otimes H^{1}\left(D_{p}, \mathbb{C}\right)_{\zeta_{p}^{p-i}}\right)\right. \\
=H^{1,0}\left(C_{1}\right)_{\zeta_{p}^{\bar{h}}} \otimes H^{1,0}\left(D_{p}\right)_{\zeta_{p}^{p-\bar{h}}} \oplus \sum_{i=1}^{(p-1) / 2}\left(H^{1,0}\left(C_{1}\right)_{\zeta_{p}^{i}} \otimes H^{0,1}\left(D_{p}\right)_{\zeta_{p}^{p-i}}\right) \\
\oplus H^{0,1}\left(C_{1}\right)_{\zeta_{p}^{p-\bar{h}}} \otimes H^{0,1}\left(D_{p}\right)_{\zeta_{p}^{\bar{h}}} \oplus \sum_{i=(p+1) / 2}^{(p-1)}\left(H^{0,1}\left(C_{1}\right)_{\zeta_{p}^{i}} \otimes H^{1,0}\left(D_{p}\right)_{\zeta_{p}^{p-i}}\right) .
\end{aligned}
$$

Now we consider the splitting given by the choice of $\Sigma=\left\{\zeta_{p}, \ldots, \zeta_{p}^{(p-1) / 2}\right\}$ and we recall that the action of $\mathbf{g}_{S}$ on $T_{S}$ is induced by the action of $\delta_{p}$ on $D_{p}$. So $\left(\sum_{i=(p-1) / 2}^{p-1}\left(H^{0,1}\left(C_{1}\right)_{\zeta_{p}^{i}} \otimes H^{1,0}\left(D_{p}\right)_{\zeta_{p}^{p-i}}\right)\right)_{\bar{\Sigma}}=0$. Hence,

$$
\begin{aligned}
\left(\left(H^{1}(C, \mathbb{Q}) \otimes_{\mathbb{Q}} H^{1}\left(D_{p}, \mathbb{Q}\right)\right)^{\mathbf{g}_{1} \times \delta_{p}}\right)_{1 / 2}^{1,0}= & \left(H^{1,0}\left(C_{1}\right)_{\zeta_{p}^{h}} \otimes H^{1,0}\left(D_{p}\right)_{\zeta_{p}^{p-h}}\right) \\
& \oplus\left(\sum_{i=1}^{(p-1) / 2} H^{1,0}\left(C_{1}\right)_{\zeta_{p}^{i}} \otimes H^{0,1}\left(D_{p}\right)_{\zeta_{p}^{p-i}}\right) .
\end{aligned}
$$

Since $H^{1,0}\left(D_{p}\right)_{\zeta_{p}^{i}} \simeq \mathbb{C}$ if $i \leq(p-1) / 2$, we obtain

$$
\left(\left(H^{1}(C, \mathbb{Q}) \otimes_{\mathbb{Q}} H^{1}\left(D_{p}, \mathbb{Q}\right)\right)^{\mathbf{g}_{1} \times \delta_{p}}\right)_{1 / 2}^{1,0} \simeq H^{1,0}\left(C_{1}\right)_{\zeta_{p}^{h}} \oplus \sum_{i=1}^{(p-1) / 2} H^{1,0}\left(C_{1}\right)_{\zeta_{p}^{i}}=H^{1,0}\left(C_{1}\right) .
$$


By conjugacy,

$$
\left(\left(H^{1}\left(C_{1}, \mathbb{Q}\right) \otimes_{\mathbb{Q}} H^{1}\left(D_{p}, \mathbb{Q}\right)\right)^{\mathbf{g}_{1} \times \delta_{p}}\right)_{1 / 2}^{0,1}=H^{0,1}\left(C_{1}\right) .
$$

Substituting in $(8.1)$, we obtain $\left(T_{S} \otimes \mathbb{Q}\right)_{1 / 2} \simeq H^{1}\left(C_{1}, \mathbb{C}\right)$ as Hodge structure.

In particular $\operatorname{rk}\left(T_{S} \otimes \mathbb{Q}\right)=2 g\left(C_{1}\right)$ and by the computation of the moduli of the K3 surface $S$ with a non-symplectic automorphism of order $p$, it follows $m=2 g\left(C_{1}\right) /(p-1)-1$.

Remark 8.2. The eigenspaces decomposition (for the action of $\mathbf{g}_{1}$ ) of $H^{1}\left(C_{1}, \mathbb{Q}\right.$ ) splits this space in $(p-1)$ equidimensional subspaces and thus $(p-1)\left(\operatorname{dim}\left(H^{1}\left(C_{1}\right)_{\zeta_{p}^{j}}\right)\right)$ $=2 g\left(C_{1}\right)$ for every $j \in\{1, \ldots, p-1\}$. By the previous proposition we have $\operatorname{dim}\left(H^{1}\left(C_{1}\right)_{\zeta_{p}^{j}}\right)=2 g\left(C_{1}\right) /(p-1)=m+1$ for every $j \in\{1, \ldots, p-1\}$. Moreover, in the proof of the previous proposition we saw that there exists a unique value $\bar{h}$ such that $\bar{h}>p-1$ and $H^{1,0}\left(C_{1}\right)_{\zeta_{p}^{\bar{h}}}$ is non zero. In particular $\operatorname{dim}\left(H^{1,0}\left(C_{1}\right)_{\zeta_{p}^{\bar{h}}}\right)=1$, because $p_{g}(S)=1$. So

$$
\begin{aligned}
m & =\operatorname{dim}\left(H^{1}\left(C_{1}\right)_{\zeta_{p}^{\bar{h}}}\right)-1=\operatorname{dim}\left(H^{1,0}\left(C_{1}\right)_{\zeta_{p}^{\bar{h}}} \oplus H^{0,1}\left(C_{1}\right)_{\zeta_{p}^{\bar{h}}}\right)-1 \\
& =\operatorname{dim}\left(H^{1,0}\left(C_{1}\right)_{\zeta_{p}^{p-\bar{h}}}\right)=\alpha_{\bar{h}} .
\end{aligned}
$$

Remark 8.3. In case $S$ is the minimal model of $\left(C_{1} \times D_{p}\right) /\left(\mathbf{g}_{1} \times \tau_{p}\right)$ one can obtain a result similar to the one of Proposition 8.1: the half twist of $T_{S} \otimes \mathbb{Q}$ is a sub-Hodge structure of $H^{1}\left(C_{1}, \mathbb{Q}\right)$ and in fact the one of $\sum_{i=1}^{p-1} H^{1}\left(C_{1}, \mathbb{C}\right)_{-\zeta_{p}^{i}}$. An explicit example is given in Section 3.11 of [17].

The variation of the period of $S$ is described by the Picard-Fuchs equation of $\omega_{S}$, and so by the Picard-Fuchs equation of certain holomorphic 1-form on $C_{1}$. In particular if $C_{1}$ varies in a 1-dimensional family, then it admits an equation of type $y^{N}=t^{a}(t-1)^{b}(t-\lambda)^{b}$. The forms of these curves and their PicardFuchs equations are described in Section 2.5 of [14], and this immediately gives the Picard-Fuchs equations of $S$.

For example the Picard-Fuchs equation of the 1-dimensional family $\mathcal{M}_{(10,0,2)}^{5}$ is the Picard-Fuchs equation of the 1-holomorphic form $\omega_{C}$ of $C_{1}$ such that $\mathbf{g}_{1}\left(\omega_{C}\right)=$ $\zeta_{p}^{3} \omega_{C}$. Since an equation for $C_{1}$ is $y^{5}=t(t-1)(t-\lambda)$, the holomorphic form we are interested in is $\omega_{1}:=d t / y^{2}$ and its Picard Fuchs equation is

$$
\lambda(1-\lambda) \frac{\partial^{2}}{\partial \lambda^{2}}+\left(\frac{4}{5}-\frac{8}{5} \lambda\right) \frac{\partial}{\partial \lambda}-\frac{2}{25}=\frac{2}{5} \frac{t(t-1)}{(t-\lambda) y^{2}} .
$$

\section{References}

[1] Artebani, M. And Sarti, A.: Non-symplectic automorphisms of order 3 on $K 3$ surfaces. Math. Ann. 342 (2008), no. 4, 903-921.

[2] Artebani, M., Sarti, A. And Taki S.: K3 surfaces with non-symplectic automorphisms of prime order. Math. Z. 268 (2011), no. 1-2, 507-533. 
[3] Barth, W.P., Hulek, K., Peters, C. A. M. and van de Ven, A.: Compact complex surfaces. A Series of Modern Surveys in Mathematics 4, Springer-Verlag, Berlin, 2004.

[4] Bauer, I., Catanese, F., Grunewald, F. and Pignatelli, R.: Quotients of products of curves, new surfaces with $p_{g}=0$ and their fundamental groups. Amer. J. Math. 134 (2012), no. 4, 993-1049.

[5] Bauer, I. and Pignatelli, R.: The classification of minimal product-quotient surfaces with $p_{g}=0$. Math. Comp. 81 (2012), no. 280, 2389-2418.

[6] Catanese, F.: Fibred surfaces, varieties isogenous to a product and related moduli spaces. Amer. J. Math. 122 (2000), no. 1, 1-44.

[7] Chevalley, C., Weil, A. And Heche, E.: Über das Verhalten der Integrale 1. Gattung bei Automorphismen des Funktionenkörpers. Abh. Math. Sem. Univ. Hamburg 10 (1934), no. 1, 358-361.

[8] Dillies, J.: On some order 6 non-symplectic automorphisms of elliptic K3 surfaces. Albanian J. Math. 6 (2012), no. 2, 103-114.

[9] Dolgachev, I.: Weighted projective varieties. In Group actions and vector fields (Vancouver, BC, 1981), 34-71. Lecture Notes in Math. 956, Springer, Berlin, 1982.

[10] Dolgachev, I., van Geemen, B. And Kondō, S.: A complex ball uniformization of the moduli space of cubic surfaces via periods of K3 surfaces. J. Reine Angew. Math. 588 (2005), 99-148.

[11] Dolgachev, I. V. And Kondō, S.: Moduli of K3 surfaces and complex ball quotients. In Arithmetic and geometry around hypergeometric functions, 43-100. Progr. Math. 260, Birkhäuser, Basel, 2007.

[12] Iano-Fletcher, A. R.: Working with weighted complete intersections. In Explicit birational geometry of 3-folds, 101-173. London Math. Soc. Lecture Note Ser. 281, Cambridge Univ. Press, Cambridge, 2000.

[13] Freitag, E.: Über die Struktur der Funktionenkörper zu hyperabelschen Gruppen I. J. Reine Angew. Math. 247 (1971), 97-117.

[14] Garbagnati, A. And van Geemen, B.: The Picard-Fuchs equation of a family of Calabi-Yau threefolds without maximal unipotent monodromy. Int. Math. Res. Not. IMRN (2010), no. 16, 3134-3143.

[15] Garbagnati, A. And Sarti, A.: On symplectic and non-symplectic automorphisms on K3 surfaces. Rev. Mat. Iberoam. 29 (2013), no. 1, 135-162.

[16] van Geemen, B.: Half twists of Hodge structures of CM-type. J. Math. Soc. Japan 53 (2001), no. 4, 813-833.

[17] van Geemen, B.: Projective models of Picard modular varieties. In Classification of irregular varieties (Trento, 1990), 68-99. Lecture Notes in Math. 1515, Springer, Berlin, 1992.

[18] Griffiths, P. And Harris, J.: Principles of algebraic geometry. Pure and Applied Mathematics, Wiley Interscience, New York, 1978.

[19] Harvey, W. J.: On branch loci in Teichmüller space. Trans. Amer. Math. Soc. 153 (1971), 387-399.

[20] Kondō, S.: Automorphisms of algebraic K3 surfaces which act trivially on Picard groups. J. Math. Soc. Japan 44 (1992), no. 1, 75-98.

[21] Kondō, S.: The moduli space of 5 points on $\mathbb{P}^{1}$ and K3 surfaces. In Arithmetic and geometry around hypergeometric functions, 189-206. Progr. Math. 260, Birkhäuser, Basel, 2007. 
[22] Magma database of small groups. http://magma.maths.usyd.edu.au/magma/ htmlhelp/text $404 . \mathrm{htm}$.

[23] Matsumoto, K. and Terasoma, T.: Theta constants associated to cubic threefolds. J. Algebraic Geom. 12 (2003), no. 4, 741-775.

[24] Miranda, R.: Algebraic curves and Riemann surfaces. Graduate Studies in Mathematics 5, American Mathematical Society, Providence, RI, 1995.

[25] Miranda, R.: The basic theory of elliptic surfaces. Dottorato di Ricerca in Matematica, Dipartimento di Matematica dell' Università di Pisa, ETS Editrice Pisa, 1989. Available at: http://www.math.colostate.edu/ miranda/BTES-Miranda.pdf.

[26] Mistretta, E. And Polizzi, F.: Standard isotrivial fibrations with $p_{g}=q=1$. II. J. Pure Appl. Algebra 214 (2010), no. 4, 344-369.

[27] Polizzi, F.: Numerical properties of isotrivial fibrations. Geom. Dedicata 147 (2010), 323-355.

[28] Oguiso, K. And Zhang, D-Q.: On Vorontsov's theorem on K3 surfaces with nonsymplectic group actions. Proc. Amer. Math. Soc. 128 (2000), no. 6, 1571-1580.

[29] Oguiso, K. and Zhang, D-Q.: K3 surfaces with order 11 automorphisms. Pure Appl. Math. Q. 7 (2011), no. 4, 1657-1673.

[30] Oguiso, K. And Zhang, D-Q.: K3 surfaces with order five automorphisms. J. Math. Kyoto Univ. 38 (1998), no. 3, 419-438.

[31] ReID, M.: Canonical 3-folds. In Journées de Géometrie Algébrique d'Angers, Juillet 1979/Algebaic Geometry, Angers, 1979, 273-310. Sijthoff \& Noordhoff, Alphen aan den Rijn-Germantown, Md., 1980.

[32] Rohde, J. C.: Cyclic coverings, Calabi-Yau manifolds and complex multiplication. Lecture Notes in Mathematics 1975, Springer-Verlag, Berlin, 2009.

[33] Serrano, F.: Isotrivial fibred surfaces. Ann. Mat. Pura Appl. (4) 171 (1996), 63-81.

[34] Shioda, T. And Inose, H.: On singular K3 surfaces. In Complex analysis and algebraic geometry, 119-136. Iwanami Shoten, Tokyo, 1977.

[35] TAKI, S.: Classification of non-symplectic automorphisms of order 3 on K3 surfaces. Math. Nachr. 284 (2011), no. 1, 124-135.

Received December 18, 2013; revised December 11, 2014.

Alice Garbagnati: Dipartimento di Matematica "Federigo Enriques", Università degli Studi di Milano, Via Saldini 50, 20133 Milano, Italy.

E-mail: alice.garbagnati@unimi.it

Matteo Penegini: Dipartimento di Matematica "Federigo Enriques", Università degli Studi di Milano, Via Saldini 50, 20133 Milano, Italy.

E-mail: matteo.penegini@unimi.it

Both authors are partially supported by PRIN 2010-2011 "Geometria delle varietà algebriche". 